\title{
ON THE ORBIT SPACE OF AN IRREDUCIBLE REPRESENTATION OF THE SPECIAL UNITARY GROUP
}

\author{
O. G. STYRT
}

\begin{abstract}
We prove that the quotient of an irreducible representation of a special unitary group of rank greater than 1 cannot be a smooth manifold.
\end{abstract}

\section{$\S 1$ INTRODUCTION}

This paper is a direct continuation of [1] and [2]. First, we recall three basic definitions playing a key role in those papers.

Definition. A continuous map between smooth manifolds is said to be piecewise smooth if it sends any smooth submanifold to a finite union of smooth submanifolds.

In particular, any proper smooth map between smooth manifolds is piecewise smooth.

Consider a differentiable action of a compact Lie group $G$ on a smooth manifold $M$.

Definition. We shall say that the quotient $M / G$ is piecewise diffeomorphic to a smooth manifold $M^{\prime}$ if the topological quotient $M / G$ is homeomorphic to $M^{\prime}$ and the quotient map $M \rightarrow M^{\prime}$ is piecewise smooth.

Definition. We shall say that the quotient $M / G$ is a smooth manifold if it is piecewise diffeomorphic to a smooth manifold.

Let us now describe our problem.

Let $V$ be a real vector space and $G \subset \mathbf{G L}(V)$ a compact linear group. As in 1 and [2, we want to know if the quotient $V / G$ is a topological manifold and also if it is a smooth manifold. Following [1] and [2, we shall call a topological manifold simply a manifold.

Let $V_{\mathbb{C}}$ denote the complex space $V \otimes \mathbb{C}, \mathfrak{g}$ the linear Lie algebra Lie $G \subset \mathfrak{g l}(V)$, and $\mathfrak{g}_{\mathbb{C}}$ the complex linear Lie algebra $\mathfrak{g} \otimes \mathbb{C} \subset \mathfrak{g l}\left(V_{\mathbb{C}}\right)$.

At the present moment, the following two cases have been dealt with: $[\mathfrak{g}, \mathfrak{g}]=0$ (see [1]) and $\mathfrak{g} \cong \mathfrak{s u}_{2}$ (see [2]). In this paper, we consider the case when $\mathfrak{g} \cong \mathfrak{s u}_{r+1}$, where $r=\operatorname{rk} \mathfrak{g}>1$ and the linear Lie algebra $\mathfrak{g} \subset \mathfrak{g l}(V)$ is irreducible.

Let $R^{\prime}$ denote the representation of a complex reductive Lie group dual to the representation $R$.

When speaking of indecomposable root systems we shall use the numbering of the simple roots adopted in [3, Table 1] and [4, Table 1], where $\left(i_{1}, \ldots, i_{m}\right)$ denotes the subset of simple roots indexed by $i_{1}, \ldots, i_{m}, \varphi_{i}$ denotes the fundamental weight indexed by $i$, and $\varphi(\mathfrak{h})$ is the linear algebra corresponding to the irreducible representation of the complex simple algebra $\mathfrak{h}$ with highest weight $\varphi$.

The space $V$ admits a $G$-invariant scalar product and hence may (and will) be considered as a Euclidean space on which $G$ acts by orthogonal operators. Thus, $G \subset \mathbf{O}(V)$.

Let $\widetilde{R}$ be the tautological representation $\mathfrak{g}_{\mathbb{C}}: V_{\mathbb{C}}$. We may have the following cases:

2010 Mathematics Subject Classification. Primary 22E46; Secondary 17B10, 17B20, $17 \mathrm{~B} 45$.

Key words and phrases. Lie group, topological action quotient. 
1) $\widetilde{R}=R$, where $R$ is a faithful irreducible representation; and

2) $\widetilde{R}=R+R^{\prime}$, where $R$ is a faithful irreducible representation.

In the latter case, $V$ admits a $\mathfrak{g}$-invariant complex structure, which naturally yields a (complex) representation $\mathfrak{g}_{\mathbb{C}}: V$ isomorphic to $R$.

Suppose now that $\mathfrak{g} \cong \mathfrak{s u}_{r+1}$, where $r=\operatorname{rk} \mathfrak{g}>1$, and the linear Lie algebra $\mathfrak{g} \subset \mathfrak{g l}(V)$ is irreducible.

In this paper we shall prove the following Theorems 1.1 and 1.2

Theorem 1.1. The quotient $V / G$ can be a smooth manifold only in the following cases:

1) the representation $\widetilde{R}$ of $\mathfrak{g}_{\mathbb{C}}$ coincides with the representation $R$ and is isomorphic to one of the representations ad, $R_{\varphi_{2}}(r=3), R_{2 \varphi_{2}}(r=3)$, and $R_{\varphi_{4}}(r=7)$;

2) $\widetilde{R}=R+R^{\prime}$ and the representation $R$ is isomorphic (up to an outer automorphism of the algebra $\left.\mathfrak{g}_{\mathbb{C}}\right)$ to one of the representations $R_{\varphi_{1}}, R_{2 \varphi_{1}}, R_{\varphi_{2}}(r>3)$, and $R_{\varphi_{3}}$ $(r=5)$.

Theorem 1.2. If $G=G^{0}$ and the representation $R$ is isomorphic (up to an outer automorphism of $\left.\mathfrak{g}_{\mathbb{C}}\right)$ to one of the representations ad, $R_{\varphi_{1}}, R_{2 \varphi_{1}}, R_{\varphi_{2}}(r>2), R_{2 \varphi_{2}}$ $(r=3), R_{\varphi_{3}}(r=5)$, and $R_{\varphi_{4}}(r=7)$, then $V / G$ is not a manifold.

Corollary 1.3. If $G=G^{0}$, then the quotient $V / G$ is not a smooth manifold.

In 92 , we introduce some notation used later in the text, and also prove some auxiliary results. In 43 we prove Theorem 1.1 and in $\$$, we prove Theorem 1.2 .

\section{$\S 2$. Notation and useful faCts}

In this section we mention a number of useful notation and results, including the ones taken from [1, 2], and [5] (all new assertions have proofs).

For a linear representation of a Lie group $G$ (respectively, a Lie algebra $\mathfrak{g}$ ) in the space $V$, the stabilizer (respectively, the isotropy subalgebra) of a vector $v \in V$ will be denoted by $G_{v}$ (respectively, by $\mathfrak{g}_{v}$ ).

In [5], for each indecomposable simple root system $\Pi$ a certain subset $\partial \Pi \subset \Pi$ was defined.

All indecomposable simple root systems $\Pi$ such that $\partial \Pi \neq \Pi$ and $\partial \Pi \subset \Pi$ are listed in [5. $\S 4$, Table 1]. For example, if $\Pi \cong A_{r}$, then

$$
\partial \Pi= \begin{cases}(1,2, r-1, r), & r \geqslant 6, \\ \Pi, & r<6 .\end{cases}
$$

2.1. Representations of compact groups. Suppose we have a Euclidean space $V$, a compact Lie group $G$ with Lie algebra $\mathfrak{g}$, a linear representation $G \rightarrow \mathbf{O}(V)$, and its differential - a representation $\mathfrak{g}: V$.

For any vector $v \in V$, let $N_{v}$ denote the subspace $(\mathfrak{g} v)^{\perp} \subset V$. Clearly $\mathfrak{g}_{v}=\operatorname{Lie} G_{v}$ and $G_{v} N_{v}=N_{v}(v \in V)$.

Lemma 2.1. If $V / G$ is a (smooth) manifold, then each quotient $N_{v} / G_{v}, v \in V$, is also a (smooth) manifold.

Proof. See Lemma 2.3 in [2, §2].

Lemma 2.2. Suppose $\operatorname{dim} \mathfrak{g}=1$.

1) If $V / G$ is a manifold, then $\operatorname{dim}(\xi V) \neq 2$ for any vector $\xi \in \mathfrak{g}$.

2) If $V / G$ is a smooth manifold, then $\operatorname{dim}(\xi V) \leqslant 6$ for any vector $\xi \in \mathfrak{g}$.

Proof. See Corollary 2.3 in [2, §2]. 
Lemma 2.3. If $\mathfrak{g} \cong \mathfrak{s u}_{2}$ and $V / G$ is a smooth manifold, then the sum of the integer parts of halves of the dimensions of all irreducible components of the representation $\mathfrak{g}: V$ (with their multiplicities) is less than or equal to 4.

Proof. See Theorem 1.1 in [2, §1].

Corollary 2.4. If $\mathfrak{g} \cong \mathfrak{s u}_{2}$ and $V / G$ is a smooth manifold, then $\operatorname{dim}(\xi V) \leqslant 8$ for any $\xi \in \mathfrak{g}$.

Corollary 2.5. If $\mathrm{rk} \mathfrak{g}=1$ and $V / G$ is a smooth manifold, then $\operatorname{dim}(\xi V) \leqslant \operatorname{dim} \mathfrak{g}+5$ for any $\xi \in \mathfrak{g}$.

Proof. Follows from Lemma 2.2 and Corollary 2.4 .

Corollary 2.6. If $\mathrm{rk} \mathfrak{g}=1$ and $V / G$ is a smooth manifold, then $\operatorname{dim}(\xi V) \leqslant \operatorname{dim}[\xi, \mathfrak{g}]+6$ for any nonzero vector $\xi \in \mathfrak{g}$.

Proof. Since $\mathrm{rk} \mathfrak{g}=1$, we have $\operatorname{Ker}(\operatorname{ad} \xi)=\mathbb{R} \xi$ for any nonzero vector $\xi \in \mathfrak{g}$, whence $\operatorname{dim}[\xi, \mathfrak{g}]=\operatorname{dim} \mathfrak{g}-1$. Now use Corollary 2.5 .

Lemma 2.7. Suppose that $V / G$ is a smooth manifold. Then $\operatorname{dim}(\xi V) \leqslant \operatorname{dim}[\xi, \mathfrak{g}]+6$ for any $v \in V$ such that $\mathrm{rk} \mathfrak{g}_{v}=1$ and for any nonzero $\xi \in \mathfrak{g}_{v}$.

Proof. By Lemma 2.1, $N_{v} / G_{v}$ is a smooth manifold. Applying Corollary 2.6 to the representation $G_{v}: N_{v}$, we have $\operatorname{dim}\left(\xi N_{v}\right) \leqslant \operatorname{dim}\left[\xi, \mathfrak{g}_{v}\right]+6$ and, therefore,

$$
\begin{aligned}
\operatorname{dim}(\xi V) & =\operatorname{dim}(\xi(\mathfrak{g} v))+\operatorname{dim}\left(\xi N_{v}\right) \\
& \leqslant \operatorname{dim}(\xi(\mathfrak{g} v))+\operatorname{dim}\left[\xi, \mathfrak{g}_{v}\right]+6=\operatorname{dim}[\xi, \mathfrak{g}]+6 .
\end{aligned}
$$

Lemma 2.8. Suppose that $V / G$ is a manifold. Then $\operatorname{dim}(\xi V) \neq \operatorname{dim}[\xi, \mathfrak{g}]+2$ for any $v \in V$ such that $\operatorname{dim} \mathfrak{g}_{v}=1$ and for any $\xi \in \mathfrak{g}_{v}$.

Proof. By Lemma 2.1 $N_{v} / G_{v}$ is a manifold. Applying Lemma 2.2 to the representation $G_{v}: N_{v}$, we have $\operatorname{dim}\left(\xi N_{v}\right) \neq 2$. Furthermore, $\left[\xi, \mathfrak{g}_{v}\right]=0$ and $\operatorname{dim}(\xi(\mathfrak{g} v))=\operatorname{dim}[\xi, \mathfrak{g}]$, whence

$$
\begin{aligned}
\operatorname{dim}(\xi V) & =\operatorname{dim}(\xi(\mathfrak{g} v))+\operatorname{dim}\left(\xi N_{v}\right) \\
& =\operatorname{dim}[\xi, \mathfrak{g}]+\operatorname{dim}\left(\xi N_{v}\right) \neq \operatorname{dim}[\xi, \mathfrak{g}]+2 .
\end{aligned}
$$

Lemma 2.9. Let $v_{1}, \ldots, v_{k} \in V$ be arbitrary vectors. If $\left(\mathfrak{g} v_{i}, v_{j}\right)=0$ for all $i, j \in$ $\{1, \ldots, k\}, i \neq j$, then, in the subspace $\left\langle v_{1}, \ldots, v_{k}\right\rangle \subset V$, there is a vector with stabilizer $\bigcap_{i=1}^{k} G_{v_{i}} \subset G$.

Proof. We induct on $k \in \mathbb{N}$.

When $k=1$ there is nothing to prove.

Now we prove the lemma for $k \in \mathbb{N} \backslash\{1\}$ assuming that it holds for $k-1 \in \mathbb{N}$.

By the induction assumption, there is $v \in\left\langle v_{1}, \ldots, v_{k-1}\right\rangle$ with stabilizer

$$
G_{v}=\bigcap_{i=1}^{k-1} G_{v_{i}} \subset G .
$$

By assumption, $\left(\mathfrak{g} v_{i}, v_{k}\right)=0(i=1, \ldots, k-1)$, and therefore $\left(\mathfrak{g} v, v_{k}\right)=0, v_{k} \in N_{v}$. Hence, for some $v^{\prime}=v+\varepsilon v_{k} \in\left\langle v_{1}, \ldots, v_{k}\right\rangle \cap N_{v}, \varepsilon \in \mathbb{R}_{>0}$, we have $G_{v^{\prime}} \subset G_{v}$, and, as a consequence,

$$
G_{v^{\prime}}=G_{v} \cap G_{\varepsilon v_{k}}=\bigcap_{i=1}^{k} G_{v_{i}} .
$$


Assertion 2.10. If $G=\mathbf{S U}_{m} \times \mathbf{S U}_{m}$ and $V=\mathbb{R}^{d} \oplus \mathfrak{g l}_{m}(\mathbb{C})(d \geqslant 0, m>1)$, and the action $G: V$ is given by $\left(g_{1}, g_{2}\right):(x, y) \rightarrow\left(x, g_{1} y g_{2}^{-1}\right)\left(g_{1}, g_{2} \in \mathbf{S U}_{m}, x \in \mathbb{R}^{d}, y \in \mathfrak{g l}_{m}(\mathbb{C})\right)$, then $V / G$ is not a manifold.

Proof. Set $v:=E \in \mathfrak{g l}_{m}(\mathbb{C}) \subset V$. It is easy to see that

$$
G_{v}=\left\{(g, g): g \in \mathbf{S U}_{m}\right\} \subset G, \quad \mathfrak{g} v=\mathfrak{s u}_{m} \subset \mathfrak{g l}_{m}(\mathbb{C}) \subset V
$$

and

$$
V=\mathbb{R}^{d} \oplus(\mathbb{C} E) \oplus \mathfrak{s u _ { m }} \oplus\left(i \cdot \mathfrak{s u} \mathfrak{u}_{m}\right) .
$$

This implies (since the groups $\mathbf{S U}_{m}$ and $G_{v}$ are naturally isomorphic) that the representation $G_{v}: N_{v}$ is isomorphic to the direct sum of the trivial action $G_{v}: \mathbb{R}^{d+2}$ and the adjoint representation $\mathbf{S U}_{m}: \mathfrak{s u}_{m}$. Hence,

$$
\frac{N_{v}}{G_{v}} \cong \mathbb{R}^{d+2} \times \mathbb{R}_{\geqslant 0}^{m-1} \cong \mathbb{R}_{\geqslant 0}^{m+d+1}
$$

Thus, the quotient $N_{v} / G_{v}$ is not a manifold and, by Lemma 2.1, neither is the quotient $V / G$.

Suppose that the representation $\mathfrak{g}: V$ is irreducible.

Let $\mathfrak{g}_{\mathbb{C}}$ denote the complex reductive Lie algebra $\underset{\mathfrak{V}}{\mathfrak{V}} \mathbb{C}$.

It is easy to see that there are a complex space $\widetilde{V}$ and an irreducible representation $\mathfrak{g}_{\mathbb{C}}: \widetilde{V}$ such that the representation $\mathfrak{g}: V$ is either the realification or a real form of the representation $\mathfrak{g}: \widetilde{V}$.

Set $\delta:=1 \in \mathbb{R}$ when $\widetilde{V}=V \otimes \mathbb{C}$ and $\delta:=2 \in \mathbb{R}$ when $\widetilde{V}=V$.

We now fix a maximal commutative subalgebra $\mathfrak{t}$ of $\mathfrak{g}$ and a Cartan subalgebra $\mathfrak{t}_{\mathbb{C}}:=$ $\mathfrak{t} \otimes \mathbb{C}$ of $\mathfrak{g}_{\mathbb{C}}$. We then have a root system $\Delta \subset \mathfrak{t}_{\mathbb{C}}^{*}$ and the corresponding Weyl group $W \subset \mathbf{G L}\left(\mathfrak{t}_{\mathbb{C}}^{*}\right)$. We also fix a simple root system $\Pi \subset \Delta \subset \mathfrak{t}_{\mathbb{C}}^{*}$.

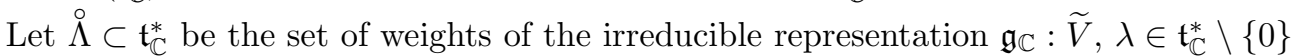
the highest weight of this representation relative to the simple root system $\Pi \subset \Delta \subset \mathfrak{t}_{\mathbb{C}}^{*}$,

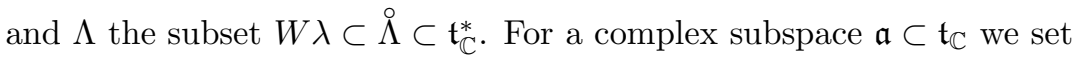

$$
\mathfrak{g}_{\mathbb{C}}(\mathfrak{a}):=\mathfrak{a} \oplus\left(\underset{\substack{\alpha \in \Delta, h_{\alpha} \in \mathfrak{a}}}{\bigoplus}\left(\mathfrak{g}_{\mathbb{C}}\right)_{\alpha}\right) \subset \mathfrak{g}_{\mathbb{C}} .
$$

Finally, for a subset $\Omega \subset \mathfrak{t}_{\mathbb{C}}^{*}$, we set

$$
\mathfrak{t}_{\mathbb{C}}^{\Omega}:=\bigcap_{\omega \in \Omega}(\operatorname{Ker} \omega) \subset \mathfrak{t}_{\mathbb{C}} \quad \text { and } \quad \mathfrak{g}^{\Omega}:=\mathfrak{g}_{\mathbb{C}}\left(\mathfrak{t}_{\mathbb{C}}^{\Omega}\right) \cap \mathfrak{g} \subset \mathfrak{g}
$$

We then have

$$
\mathfrak{g}^{\Omega_{1} \cup \Omega_{2}}=\mathfrak{g}^{\Omega_{1}} \cap \mathfrak{g}^{\Omega_{2}} \subset \mathfrak{g} \quad\left(\Omega_{1}, \Omega_{2} \subset \mathfrak{t}_{\mathbb{C}}^{*}\right) .
$$

Consider an arbitrary weight $\lambda^{\prime} \in \Lambda$.

For any vector $v \neq 0$ in the (nontrivial) subspace $\widetilde{V}_{\lambda^{\prime}} \subset \widetilde{V}$, the subalgebra $\left(\mathfrak{g}_{\mathbb{C}}\right)_{v}$ of $\mathfrak{g}_{\mathbb{C}}$ coincides with the direct sum of all subspaces $\operatorname{Ker} \lambda^{\prime} \subset \mathfrak{t}_{\mathbb{C}}$ and $\left(\mathfrak{g}_{\mathbb{C}}\right)_{\alpha}\left(\alpha \in \Delta,\left\langle\lambda^{\prime} \mid \alpha\right\rangle \geqslant 0\right)$, and therefore $\mathfrak{g}_{v}=\mathfrak{g}^{\left\{\lambda^{\prime}\right\}} \subset \mathfrak{g}$.

If $\widetilde{V}=V \otimes \mathbb{C}$ and $2 \lambda^{\prime} \notin \Delta \cup(\Delta+\Delta)$, then $-\lambda^{\prime} \in \Lambda$ and we also have

$$
\left(\mathfrak{g}_{\mathbb{C}} \widetilde{V}_{\lambda^{\prime}}\right) \cap\left(\mathfrak{g}_{\mathbb{C}} \widetilde{V}_{-\lambda^{\prime}}\right)=0, \quad\left(\mathfrak{g} \widetilde{V}_{\lambda^{\prime}}\right) \cap\left(\mathfrak{g} \widetilde{V}_{-\lambda^{\prime}}\right)=0 .
$$

Therefore, for any nonzero vector $v$ of the (nontrivial) subspace $\left(\widetilde{V}_{\lambda^{\prime}} \oplus \widetilde{V}_{-\lambda^{\prime}}\right) \cap V \subset V$, we have $\mathfrak{g}_{v}=\mathfrak{g}^{\left\{\lambda^{\prime}\right\}} \subset \mathfrak{g}$.

This and Lemma 2.9 imply the following lemma. 
Lemma 2.11. Let $\Omega \subset \Lambda$ be a subset such that $(\Omega-\Omega) \cap \Delta=\varnothing$. Suppose that one of the following conditions is satisfied: 1) $\delta=2$; 2) $\delta=1,2 \lambda \notin \Delta \cup(\Delta+\Delta)$ and $(\Omega+\Omega) \cap \Delta=\varnothing$. Then there is a vector $v \in V$ such that $\mathfrak{g}_{v}=\mathfrak{g}^{\Omega} \subset \mathfrak{g}$.

Let $\|\cdot\|$ denote the order of a subset of the set $\Lambda \subset \mathfrak{t}_{\mathbb{C}}^{*}$ of weights with multiplicities of the representation $\mathfrak{g}_{\mathbb{C}}: \widetilde{V}$.

Lemma 2.12. Suppose that $V / G$ is a smooth manifold. If $v \in V, \operatorname{rk} \mathfrak{g}_{v}=1$, and $\xi \in$ $\left(\mathfrak{g}_{v} \cap \mathfrak{t}\right) \backslash\{0\}$, then

$$
\delta \cdot\left\|\left\{\lambda^{\prime} \in \stackrel{\circ}{\Lambda}: \lambda^{\prime}(\xi) \neq 0\right\}\right\| \leqslant\|\{\alpha \in \Delta: \alpha(\xi) \neq 0\}\|+6 .
$$

Proof. By Lemma 2.7.

$$
\delta \cdot \operatorname{dim}_{\mathbb{C}}(\xi \widetilde{V})=\operatorname{dim}(\xi V) \leqslant \operatorname{dim}[\xi, \mathfrak{g}]+6=\operatorname{dim}_{\mathbb{C}}\left[\xi, \mathfrak{g}_{\mathbb{C}}\right]+6,
$$

which implies the desired assertion.

Corollary 2.13. Let $\Omega \subset \Lambda$ be an arbitrary subset. Let $H$ denote the subspace $\langle\Omega\rangle_{\mathbb{C}} \subset \mathfrak{t}_{\mathbb{C}}^{*}$. If

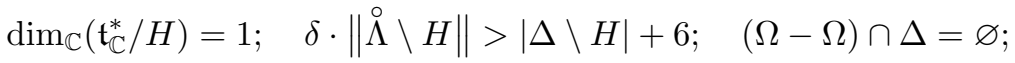

$$
\begin{aligned}
& (\delta=1) \Rightarrow((2 \lambda \notin \Delta \cup(\Delta+\Delta)) \text { and }((\Omega+\Omega) \cap \Delta=\varnothing)) \text {, }
\end{aligned}
$$

then the quotient $V / G$ is not a smooth manifold.

Proof. Follows from Lemmas 2.11 and 2.12 .

Lemma 2.14. Suppose the quotient $V / G$ is a manifold and $\stackrel{\Lambda}{\Lambda}=\Lambda$. If $v \in V, \xi \in \mathfrak{t} \backslash\{0\}$, and $\mathfrak{g}_{v}=\mathbb{R} \xi$, then

$$
\delta \cdot\left\|\left\{\lambda^{\prime} \in \Lambda: \lambda^{\prime}(\xi) \neq 0\right\}\right\| \neq\|\{\alpha \in \Delta: \alpha(\xi) \neq 0\}\|+2 .
$$

Proof. By Lemma 2.8,

$$
\delta \cdot \operatorname{dim}_{\mathbb{C}}(\xi \tilde{V})=\operatorname{dim}(\xi V) \neq \operatorname{dim}[\xi, \mathfrak{g}]+2=\operatorname{dim}_{\mathbb{C}}\left[\xi, \mathfrak{g}_{\mathbb{C}}\right]+2,
$$

which implies the desired assertion.

Corollary 2.15. Let $\Omega \subset \Lambda$ be an arbitrary subset. Let $H$ denote the subspace $\langle\Omega\rangle_{\mathbb{C}} \subset \mathfrak{t}_{\mathbb{C}}^{*}$. If

$$
\begin{gathered}
\operatorname{dim}_{\mathbb{C}}\left(\mathfrak{t}_{\mathbb{C}}^{*} / H\right)=1 ; \quad \stackrel{\Lambda}{\Lambda}=\Lambda ; \\
\delta \cdot|\Lambda \backslash H|=|\Delta \backslash H|+2 ; \quad(\Omega-\Omega) \cap \Delta=H^{\perp} \cap \Delta=\varnothing ; \\
(\delta=1) \Rightarrow \quad((2 \lambda \notin \Delta \cup(\Delta+\Delta)) \text { and }((\Omega+\Omega) \cap \Delta=\varnothing)),
\end{gathered}
$$

then $V / G$ is not a manifold.

Proof. Follows from Lemmas 2.11 and 2.14 .

\subsection{Polar representations.}

Definition. A representation of a compact Lie group $G$ in a real space $V$ is polar if there is a subspace $V^{\prime} \subset V$ such that $G V^{\prime}=V$ and $V^{\prime} \cap\left(T_{v}(G v)\right)=0\left(v \in V^{\prime}\right)$.

Lemma 2.16. The quotient space of an arbitrary nontrivial connected polar compact linear group is homeomorphic to a closed half-space.

Proof. By the results of [6] (see Theorems 2.8, 2.9, and 2.10 in $\S 2$ ), the above quotient space is homeomorphic to a quotient space of a nontrivial finite linear group generated by (real) reflections. 
Let $G$ be a connected simply-connected compact semisimple Lie group, $\mathfrak{g}$ its tangent algebra, and $\theta \in \operatorname{Aut}(G)$ a nontrivial involutive automorphism. Now consider the adjoint action $G^{\theta}: \mathfrak{g}$ and its restriction to the (clearly invariant) subspace $\mathfrak{g}^{-d \theta} \subset \mathfrak{g}$.

Lemma 2.17. The quotient $\mathfrak{g}^{-d \theta} / G^{\theta}$ is homeomorphic to a closed half-space.

Proof. By Theorem B of [7, §1], the Lie subgroup $G^{\theta} \subset G$ is connected. Since $G$ is semisimple, the representation $G^{\theta}: \mathfrak{g}^{-d \theta}$ is nontrivial. This representation is also polar (see [8, §8, 8.5-8.6]). It remains to apply Lemma 2.16.

Remark. The assertion of Lemma 2.17 also follows from the results of [9].

2.3. Combinatorial inequalities. For natural numbers $n, k, m_{1}, \ldots, m_{k}$ such that $m_{1}+$ $+\ldots+m_{k}=n$, set

$\left(\begin{array}{c}n \\ m_{1}, \ldots, m_{k}\end{array}\right):=\left(\begin{array}{c}n \\ m_{1}\end{array}\right) \cdot\left(\begin{array}{c}n-m_{1} \\ m_{2}\end{array}\right) \cdot \ldots \cdot\left(\begin{array}{c}n-m_{1}-\ldots-m_{k-2} \\ m_{k-1}\end{array}\right)=\frac{n !}{m_{1} ! \cdot \ldots \cdot m_{k} !} \in \mathbb{N}$.

Assertion 2.18. If $n, k, m_{1}, \ldots, m_{k} \in \mathbb{N}$ and $m_{1}+\ldots+m_{k}=n$, then the inequality $\left(\begin{array}{c}n \\ m_{1}, \ldots, m_{k}\end{array}\right)<n$ is possible only when $k=1$ and $m_{1}=n$.

Proof. We have $\left(\begin{array}{c}n \\ m_{1}\end{array}\right) \leqslant\left(\begin{array}{c}n \\ m_{1}, \ldots, m_{k}\end{array}\right)<n$, whence $m_{1}=n$.

Assertion 2.19. If $n, k, m_{1}, \ldots, m_{k} \in \mathbb{N}, k>1$, and $m_{1}+\ldots+m_{k}=n$, then the inequality $\left(\begin{array}{c}n \\ m_{1}, \ldots, m_{k}\end{array}\right) \leqslant 2(n-1)$ is possible only in the following cases:

1) $k=2,\left\{m_{1}, m_{2}\right\}=\{1, n-1\}$;

2) $n=4, k=2, m_{1}=m_{2}=2$.

Proof. Suppose $k>2$. Then

$$
0<m_{2}<n-m_{1}, \quad 0<m_{1}<n-m_{2} \leqslant n-1 .
$$

Therefore $\left(\begin{array}{c}n-m_{1} \\ m_{2}\end{array}\right) \geqslant n-m_{1}$, and also $\left(\begin{array}{c}n-1 \\ m_{1}\end{array}\right) \geqslant n-1$. Therefore,

$2(n-1) \geqslant\left(n m_{1}, \ldots, m_{k}\right) \geqslant\left(\begin{array}{c}n \\ m_{1}\end{array}\right) \cdot\left(n-m_{1} m_{2}\right) \geqslant\left(\begin{array}{c}n \\ m_{1}\end{array}\right) \cdot\left(n-m_{1}\right)=n \cdot\left(\begin{array}{c}n-1 \\ m_{1}\end{array}\right) \geqslant n(n-1)$,

$(n-2)(n-1) \leqslant 0, n \leqslant 2<k \leqslant m_{1}+\ldots+m_{k}=n$. This is a contradiction.

Assume now that $k=2$ and $\left\{m_{1}, m_{2}\right\} \neq\{1, n-1\}$.

We have $m_{1}, m_{2} \geqslant 2$ and $\left(\begin{array}{c}n \\ m_{1}\end{array}\right) \geqslant\left(\begin{array}{c}n \\ 2\end{array}\right)$. Moreover, $n=m_{1}+m_{2} \geqslant 4$. Therefore,

$$
2(n-1) \geqslant\left(\begin{array}{c}
n \\
m_{1}, m_{2}
\end{array}\right)=\left(\begin{array}{c}
n \\
m_{1}
\end{array}\right) \geqslant\left(\begin{array}{c}
n \\
2
\end{array}\right)=\frac{n(n-1)}{2} \geqslant \frac{4(n-1)}{2}=2(n-1),
$$

whence $n=4$, and also

$$
\left(\begin{array}{c}
n \\
m_{1}
\end{array}\right)=\left(\begin{array}{c}
n \\
2
\end{array}\right), \quad\left(\begin{array}{c}
4 \\
m_{1}
\end{array}\right)=\left(\begin{array}{l}
4 \\
2
\end{array}\right), \quad m_{1}=m_{2}=2 .
$$


2.4. The orbits of the Weyl group. Let $r>1$ be a natural number. Setting $n:=$ $r+1 \in \mathbb{N}$, we have $n \geqslant 3$.

Consider the Euclidean space $\mathbb{R}^{n}$ in which the standard basis $\left\{\varepsilon_{i}\right\}_{i=1}^{n}$ is orthonormal, an indecomposable root system $\Delta:=\left\{\varepsilon_{i}-\varepsilon_{j}: i \neq j\right\} \subset \mathbb{R}^{n}$ of type $A_{r}$, its Weyl group $W \subset$ $\mathbf{O}\left(\mathbb{R}^{n}\right)$, the Weyl chamber $C:=\left\{x \in \mathbb{R}^{n}: x_{1} \geqslant \ldots \geqslant x_{n}\right\} \subset \mathbb{R}^{n}$, and the corresponding simple root system $\Pi:=\left\{\alpha_{1}, \ldots, \alpha_{r}\right\} \subset \Delta\left(\alpha_{i}:=\varepsilon_{i}-\varepsilon_{i+1}, i=1, \ldots, r\right)$.

The lattices $P:=\{\lambda \in\langle\Delta\rangle:\langle\lambda \mid \alpha\rangle \in \mathbb{Z} \forall \alpha \in \Delta\}$ and $Q:=\langle\Delta\rangle_{\mathbb{Z}}$ in the subspace $\langle\Delta\rangle \subset \mathbb{R}^{n}$ satisfy the relations $W P=P, W Q=Q, Q \subset P$, and $|P / Q|=n<\infty$.

Let $\varphi_{1}, \ldots, \varphi_{r} \in P$ be the fundamental weights relative to the simple root system $\Pi=\left\{\alpha_{1}, \ldots, \alpha_{r}\right\} \subset \Delta$ (in the same order). Clearly $\left\{\varphi_{i}\right\}_{i=1}^{r}$ is a basis of the lattice $P \subset\langle\Delta\rangle$.

For brevity, the orbits of the action $W: P$ will be simply called orbits.

Let $\Lambda \subset P$ be an arbitrary orbit. Set $\Lambda:=\operatorname{conv}(\Lambda) \cap(\Lambda+Q) \subset P$. It is clear that:

1) $|\Lambda \cap C|=1$;

2) $\Lambda-\Lambda \subset Q$;

3) $\stackrel{\Lambda}{\supset} \Lambda$;

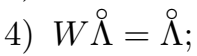

5) for any orbit $\Lambda^{\prime} \subset \stackrel{\circ}{\Lambda}$, we have $\AA^{\prime} \subset \AA$;

6) for any orbit $\Lambda^{\prime} \subset \stackrel{\circ}{\Lambda} \Lambda^{\prime} \neq \Lambda$, we have $\AA^{\prime} \cap \Lambda=\varnothing$.

Fix an orbit $\Lambda \subset P \backslash\{0\}$.

We have $\Lambda \cap C=\{\lambda\}$, where $\lambda \in(P \cap C) \backslash\{0\}$.

2.4.1. Main assertions. Let $\Pi^{\prime} \subset \Pi \subset \mathbb{R}^{n}$ be an indecomposable simple root system of order $r-2$.

It is easy to see that $H:=\left\langle\{\lambda\} \cup \Pi^{\prime}\right\rangle \subset\langle\Delta\rangle \subset \mathbb{R}^{n}$ is an $(r-1)$-dimensional subspace.

In this subsection, we prove the following Lemmas 2.20 and 2.21

Lemma 2.20. If $\lambda \notin \Delta, \lambda \neq \varphi_{1}, \ldots, \varphi_{r},|\AA \backslash H| \leqslant 4 n$, and $(-\Lambda=\Lambda) \vee(|\AA \backslash H| \leqslant 2 n)$, then either $\lambda \in\left\{2 \varphi_{1}, 2 \varphi_{r}, \varphi_{1}+\varphi_{r-1}, \varphi_{2}+\varphi_{r}\right\}$ or $(r=3)$ and $\left(\lambda=2 \varphi_{2}\right)$.

Lemma 2.21. Suppose that $r \geqslant 8, \lambda=\varphi_{j}$, where $j \in \mathbb{N}$ and $3 \leqslant j \leqslant r-2$, and also $\Pi^{\prime}=\left\{\alpha_{1}, \ldots, \alpha_{r-2}\right\} \subset \Pi$. Then $|\AA \backslash H|>4 n$.

We have $\lambda \in(\langle\Delta\rangle \cap C) \backslash\{0\}, \lambda_{1}>0>\lambda_{n}$, and therefore

$$
\left\langle\Pi^{\prime}\right\rangle=\left\{x \in\langle\Delta\rangle: x_{p}=x_{q}=0\right\}, \quad H=\left\{x \in\langle\Delta\rangle: x_{q}=c x_{p}\right\},
$$

where $(p, q) \in\{1, \ldots, n\}^{2}$ is one of the pairs $(1,2),(1, n)$ and $(n, n-1)$ (and, therefore, $\left.\lambda_{p} \neq 0\right)$, and $c$ is the number $\frac{\lambda_{q}}{\lambda_{p}} \in \mathbb{R}$.

We begin with a proof of Lemma 2.20, First we need some auxiliary results.

Let $x \in P \cap C$ be a vector.

Set $K:=\left\{x_{1}, \ldots, x_{n}\right\} \subset \mathbb{R}, k:=|K| \in \mathbb{N}, m(t):=\left|\left\{i \in\{1, \ldots, n\}: x_{i}=t\right\}\right| \in \mathbb{N}$ $(t \in K)$ and $m_{0}:=\max \{m(t)\}_{t \in K} \in \mathbb{N}$.

Proposition 2.22. Suppose that $k \geqslant 3$. Then

1) $|(W x) \backslash H| \geqslant 2 n-2$;

2) if $m_{0} \neq n-2$, then $|(W x) \backslash H| \geqslant 5(n-2)$;

3) if $k \geqslant 4$, then $|(W x) \backslash H| \geqslant 4 n$.

Proof. Set

$$
\begin{gathered}
L\left(t^{\prime}\right):=\left\{t^{\prime \prime} \in K \backslash\left\{c t^{\prime}\right\}:\left(m\left(t^{\prime}\right)=1\right) \Rightarrow\left(t^{\prime \prime} \neq t^{\prime}\right)\right\} \subset K \quad\left(t^{\prime} \in K\right), \\
L:=\left\{\left(t^{\prime}, t^{\prime \prime}\right) \in K^{2}: t^{\prime \prime} \in L\left(t^{\prime}\right)\right\} \subset K^{2}
\end{gathered}
$$


and

It is clear that

$$
K_{0}:=\{t \in K: c t \in K \backslash\{t\}, m(t)=1\} \subset K .
$$

$$
\begin{array}{rl}
\forall \tau \in L & d(\tau):=\left|\left\{y \in W x:\left(y_{p}, y_{q}\right)=\tau\right\}\right| \geqslant 1 ; \quad|(W x) \backslash H|=\sum_{\tau \in L} d(\tau) ; \\
\forall t \in K & |L(t)| \geqslant k-2 ; \\
\forall t \in K \backslash K_{0} & |L(t)|>k-2 .
\end{array}
$$

Hence

$$
\begin{gathered}
|L| \geqslant k(k-2)+\left(k-\left|K_{0}\right|\right) \geqslant k(k-2), \\
\left|K_{0}\right| \geqslant k(k-2)+k-|L|=k(k-1)-|L| .
\end{gathered}
$$

Let us show that

$$
|L| \geqslant 4 ; \quad(|L|=4) \Rightarrow\left(m_{0}=n-2\right) .
$$

Suppose $|L| \leqslant 4$. Then

$$
\begin{gathered}
k(k-2) \leqslant|L| \leqslant 4<4 \cdot(4-2), \quad k=3, \\
\left|K_{0}\right| \geqslant 6-|L| \geqslant 2, \quad|\{t \in K: m(t)=1\}| \geqslant\left|K_{0}\right| \geqslant 2 .
\end{gathered}
$$

Moreover, $n \geqslant|K|=k=3$. Hence, $m_{0}=n-2$. Now if $|L| \leqslant 3$, then $\left|K_{0}\right| \geqslant 6-|L| \geqslant$ $3=|K|, K_{0}=K$,

$\forall t \in K \quad c t \in K \backslash\{t\}, \quad c t \neq t, \quad t \neq 0, \quad c \neq 1, \quad c^{3} \neq 1, \quad c^{3} t \neq t ; \quad K \subset \mathbb{R} \backslash\{0\} ;$

$\forall t \in K \quad c t \in K \backslash\{t\} \subset \mathbb{R} \backslash\{0\}, \quad c \neq 0$,

and we thus have a bijective map $K \rightarrow K, t \rightarrow c t$, whose third power has no fixed points, whereas $|K|=3$. This is a contradiction.

We have thus established (2.2).

Let us show that

$$
l:=|\{\tau \in L: d(\tau)<n-2\}| \leqslant 2 ; \quad(l>0) \Rightarrow\left(m_{0}=n-2\right) .
$$

Fix a number $t_{0} \in K$ such that $m\left(t_{0}\right)=m_{0}$ (it does exist). We also set $L_{0}:=$ $\left\{\left(t^{\prime}, t^{\prime \prime}\right) \in K^{2}: t^{\prime}, t^{\prime \prime} \neq t_{0}, t^{\prime} \neq t^{\prime \prime}\right\} \subset K^{2}$.

Let $\left(t^{\prime}, t^{\prime \prime}\right) \in L$ be an arbitrary pair such that $d\left(t^{\prime}, t^{\prime \prime}\right)<n-2$. By Assertion 2.18 and inequalities $k \geqslant 3$, we have $k=3, K=\left\{t, t^{\prime}, t^{\prime \prime}\right\} \subset \mathbb{R}, t^{\prime} \neq t, t^{\prime \prime} \neq t, t^{\prime} \neq t^{\prime \prime}$, $m(t)=n-2>d\left(t^{\prime}, t^{\prime \prime}\right) \geqslant 1=m\left(t^{\prime}\right)=m\left(t^{\prime \prime}\right), m_{0}=n-2, t_{0}=t \in K \backslash\left\{t^{\prime}, t^{\prime \prime}\right\}$, $\left(t^{\prime}, t^{\prime \prime}\right) \in L_{0}$, where $\left|L_{0}\right|=(k-1)(k-2)=2$.

We have thus established (2.3).

It is clear that $n \geqslant|K|=k \geqslant 3$. Now, using (2.1)-(2.3), we have that

$$
\begin{aligned}
|(W x) \backslash H| \geqslant & (n-2) \cdot(|L|-l)+l=(n-2) \cdot|L|-(n-3) l \\
& =4(n-2)-2(n-3)+(n-2) \cdot(|L|-4)+(n-3) \cdot(2-l) \\
& \geqslant 4(n-2)-2(n-3)=2 n-2,
\end{aligned}
$$

and, in the case $m_{0} \neq n-2$, we also have $|L| \geqslant 5$ and $l=0$, whence

$$
|(W x) \backslash H| \geqslant(n-2) \cdot|L|-(n-3) l=(n-2) \cdot|L| \geqslant 5(n-2) .
$$

Suppose $k \geqslant 4$. Then $n \geqslant k \geqslant 4,|L| \geqslant k(k-2) \geqslant 8$, and also $m_{0} \leqslant n-(k-1)<n-2$. Therefore, $|(W x) \backslash H| \geqslant(n-2) \cdot|L| \geqslant 8(n-2)=4 n+4(n-4) \geqslant 4 n$.

This finishes the prof.

Corollary 2.23. $|\Delta \backslash H| \geqslant 2 n-2$. 
Proof. We have $x:=\varepsilon_{1}-\varepsilon_{n} \in \Delta \cap C$ and also $\left|\left\{x_{1}, \ldots, x_{n}\right\}\right|=3$. Now use Proposition 2.22 .

Proposition 2.24. For any vector $x \in(P \cap C) \backslash\{0\}$, we have the inequality $|(W x) \backslash H| \geqslant$ 2, which may become an equality only in the following cases:

1) $x_{2}=\ldots=x_{n}$;

2) $x_{1}=\ldots=x_{n-1}$;

3) $n=4, x_{1}=x_{2}, x_{3}=x_{4}$.

Proof. Since the tautological representation $W:\langle\Delta\rangle$ is irreducible, we have

$$
\begin{gathered}
\sum_{y \in W x} y=0 ; \\
\langle W x\rangle=\langle\Delta\rangle ; \\
\bigcap_{w \in W}(w H)=0 .
\end{gathered}
$$

By (2.4), if $|(W x) \backslash H| \leqslant 1$, then $W x \subset H$ and $\langle W x\rangle \subset H$, contrary to (2.5). This proves that $|(W x) \backslash H| \geqslant 2$.

Suppose that $|(W x) \backslash H|=2$.

By (2.6), there are elements $w_{1}, \ldots, w_{r} \in W$ such that $\bigcap_{i=1}^{r}\left(w_{i} H\right)=0$. We have

$$
\begin{gathered}
0 \notin W x, \quad W x=\bigcup_{i=1}^{r}\left((W x) \backslash\left(w_{i} H\right)\right), \\
|W x| \leqslant \sum_{i=1}^{r}\left|(W x) \backslash\left(w_{i} H\right)\right|=\sum_{i=1}^{r}|(W x) \backslash H|=2(n-1) .
\end{gathered}
$$

Now use Assertion 2.19.

Proposition 2.25. Let $x \in(P \cap C) \backslash\{0\}$ be a vector and $\Lambda^{\prime}$ be the orbit $W x \subset P$. Then $\left|\AA^{\prime} \backslash H\right| \geqslant 2$, and the equality $\left|\AA^{\prime} \backslash H\right|=2$ is possible only in the following cases:

1) $x_{1}-x_{2}=1, x_{2}=\ldots=x_{n}$;

2) $x_{1}=\ldots=x_{n-1}, \quad x_{n-1}-x_{n}=1$;

3) $n=4, x_{1}=x_{2}, x_{2}-x_{3}=1, x_{3}=x_{4}$.

Proof. Proposition 2.24 and the relation $\AA^{\prime} \supset \Lambda^{\prime}$ imply the inequality $\left|\AA^{\prime} \backslash H\right| \geqslant 2$, which may become an equality only in cases 1) -3) mentioned in the statement of Proposition 2.24 , provided the $\left(W\right.$-invariant) subset $\stackrel{\Lambda}{ }^{\prime} \backslash\left(\Lambda^{\prime} \sqcup\{0\}\right) \subset P$ contains no orbits, i.e., when $\stackrel{\circ}{ }^{\prime} \backslash\{0\}=\Lambda^{\prime}$.

Suppose $\left|\AA^{\prime} \backslash H\right|=2$. Then, $\stackrel{\Lambda}{ }^{\prime} \backslash\{0\}=\Lambda^{\prime}$, and one of conditions 1) - 3) from Proposition 2.24 is satisfied, whence $x \notin \Delta$. Furthermore, for any $\alpha \in \Delta$ such that $\langle x \mid \alpha\rangle>0$ we have $x-\alpha \in \stackrel{\circ}{\Lambda}^{\prime} \backslash\{0\}=\Lambda^{\prime}$, which implies that $\langle x \mid \alpha\rangle=1$. In particular, $\left\langle x \mid\left(\varepsilon_{1}-\varepsilon_{n}\right)\right\rangle=1$ and $x_{1}-x_{n}=1$.

This finishes the proof of the proposition.

Proposition 2.26. Let $x \in P \cap C$ be a vector and $\Lambda^{\prime}$ be the orbit $W x \subset P$. If $x \notin \Delta$ and $\left|\left\{x_{1}, \ldots, x_{n}\right\}\right| \geqslant 3$, then we have the inequality $\left|\AA^{\prime} \backslash H\right| \geqslant 2 n$, which may become an equality only in the following cases:

1) $x_{1}=x_{2}, x_{3}=\ldots=x_{n-1}, x_{2}-x_{3}=x_{n-1}-x_{n}=1$;

2) $x_{2}=\ldots=x_{n-2}, \quad x_{n-1}=x_{n}, x_{1}-x_{2}=x_{n-2}-x_{n-1}=1$. 
Proof. Since $\left|\left\{x_{1}, \ldots, x_{n}\right\}\right| \geqslant 3$, we have $x_{1}-x_{n}>1$. Thus, among all the pairs $\left(i_{1}, i_{2}\right) \in$ $\{1, \ldots, n\}^{2}$ such that $x_{i_{1}}-x_{i_{2}}>1$, we can choose a pair $\left(i_{1}, i_{2}\right)$ with smallest $i_{2}-i_{1}$.

Clearly, $i_{2}>i_{1}$. Moreover, if $i=i_{1}+1, \ldots, i_{2}-1$ is an arbitrary number, then $x_{i_{1}}-x_{i} \leqslant 1$ and $x_{i}-x_{i_{2}} \leqslant 1$, whereas $\left(x_{i_{1}}-x_{i}\right)+\left(x_{i}-x_{i_{2}}\right)=x_{i_{1}}-x_{i_{2}}>1$, whence $x_{i_{1}}-$ $x_{i}=x_{i}-x_{i_{2}}=1$. Thus,

$$
\begin{gathered}
x_{i_{1}}-1 \geqslant x_{i_{2}}+1 \\
\left(i_{2}-i_{1}>1\right) \Rightarrow\left(x_{i_{1}}-1=x_{i_{1}+1}=\ldots=x_{i_{2}-1}=x_{i_{2}}+1\right) .
\end{gathered}
$$

Set $\alpha:=\varepsilon_{i_{1}}-\varepsilon_{i_{2}} \in \Delta$. Since $\langle x \mid \alpha\rangle=x_{i_{1}}-x_{i_{2}}>1$ and $x \notin \Delta$ it follows that $y:=x-\alpha \in \stackrel{\circ}{ }^{\prime} \backslash\left(\Lambda^{\prime} \sqcup\{0\}\right)$. Let $\Lambda^{\prime \prime}$ denote the orbit $W y \subset P$.

We have $x \in C, x_{1} \geqslant \ldots \geqslant x_{n}$, and, according to (2.7),

$$
\begin{gathered}
x_{1} \geqslant \ldots \geqslant x_{i_{1}-1}>x_{i_{1}}-1 \geqslant x_{i_{1}+1} \geqslant \ldots \geqslant x_{i_{2}-1} \geqslant x_{i_{2}}+1>x_{i_{2}+1} \geqslant \ldots \geqslant x_{n} ; \\
\left(i_{2}-i_{1}>1\right) \Rightarrow\left(y_{i_{1}}=\ldots=y_{i_{2}}\right),
\end{gathered}
$$

$y_{1} \geqslant \ldots \geqslant y_{i_{1}-1}>y_{i_{1}} \geqslant \ldots \geqslant y_{i_{2}}>y_{i_{2}+1} \geqslant \ldots \geqslant y_{n}$. Therefore, $y \in C$. Moreover, $\stackrel{\Lambda}{ }^{\prime} \supset \Lambda^{\prime} \sqcup \stackrel{\curlywedge}{\prime \prime}^{\prime \prime}$, and therefore,

$$
\left|\AA^{\prime} \backslash H\right| \geqslant\left|\Lambda^{\prime} \backslash H\right|+\left|\AA^{\prime \prime} \backslash H\right| .
$$

Suppose $\left|\stackrel{\Lambda}{ }^{\prime} \backslash H\right| \leqslant 2 n$.

It follows from Propositions 2.22 and 2.25 that $\left|\AA^{\prime} \backslash H\right|=2 n, y_{1}=\ldots=y_{j}, y_{j+1}=$ $=\ldots=y_{n}, y_{j}-y_{j+1}=1, j \in\{1, \ldots, n-1\}$, and either $j \in\{1, n-1\}$ or $n=4$ and $j=2$.

Notice the following:

1) as we mentioned before, $y \neq 0$, and, by (2.8),$\left(i_{1}, i_{2}\right) \neq(1, n)$;

$2)$ if $i_{1}>1$, then $y_{i_{1}-1}>y_{i_{1}}, j=i_{1}-1<i_{2}-1 \leqslant n-1,(j=1) \vee((n=4)$ and $(j=2))$;

$3)$ if $i_{2}<n$, then $y_{i_{2}}>y_{i_{2}+1}, j=i_{2}>i_{1} \geqslant 1,(j=n-1) \vee((n=4)$ and $(j=2))$.

Since $i_{1}-1<i_{1}<i_{2}$, exactly one of the following equalities $i_{1}=1$ and $i_{2}=n$ holds.

We can have the following cases.

Case 1. $i_{1}>1, i_{2}=n, j=i_{1}-1$, and $j=1$.

We have $i_{1}=2, y_{1}-1=y_{2}=\ldots=y_{n}, x_{1}-1=x_{2}-1=x_{3}=\ldots=x_{n-1}=x_{n}+1$.

Case 2. $i_{1}>1, i_{2}=n, j=i_{1}-1, n=4$, and $j=2$.

We have $i_{1}=3, y_{1}=y_{2}=y_{3}+1=y_{4}+1, x_{1}=x_{2}=x_{3}=x_{4}+2$.

Case 3. $i_{1}=1, i_{2}<n, j=i_{2}$, and $j=n-1$.

We have $i_{2}=n-1, y_{1}=\ldots=y_{n-1}=y_{n}+1, x_{1}-1=x_{2}=\ldots=x_{n-2}=x_{n-1}+1=$ $x_{n}+1$.

Case 4. $i_{1}=1, i_{2}<n, j=i_{2}, n=4$, and $j=2$.

We have $i_{2}=2, y_{1}-1=y_{2}-1=y_{3}=y_{4}, x_{1}-2=x_{2}=x_{3}=x_{4}$.

Now use the fact that $\left|\left\{x_{1}, \ldots, x_{n}\right\}\right| \geqslant 3$.

Proposition 2.27. Let $x \in P \cap C$ be a vector and let $\Lambda^{\prime}$ denote the orbit $W x \subset P$. If

$$
\left|\left\{x_{1}, \ldots, x_{n}\right\}\right|=2,
$$

$x_{1}-x_{n}>1$, and $\left|\AA^{\prime} \backslash H\right| \leqslant 2 n$, then one of the following holds:

1) $x_{1}-x_{2}=2, x_{2}=\ldots=x_{n}$;

2) $x_{1}=\ldots=x_{n-1}, x_{n-1}-x_{n}=2$;

3) $n=4, x_{1}=x_{2}=1, x_{3}=x_{4}=-1$. 
Proof. By assumption, $x_{1}=\ldots=x_{j}, x_{j+1}=\ldots=x_{n}$, and $x_{j}-x_{j+1} \geqslant 2$, where $j \in\{1, \ldots, n-1\}$.

Set $\alpha:=\alpha_{j} \in \Delta$. We have $\langle x \mid \alpha\rangle=x_{j}-x_{j+1}>1$, and therefore $y:=x-\alpha \in \stackrel{\circ}{ }^{\prime} \backslash \Lambda^{\prime}$. Moreover, $x \in C, x_{1} \geqslant \ldots \geqslant x_{n}, x_{1} \geqslant \ldots \geqslant x_{j-1}>x_{j}-1 \geqslant x_{j+1}+1>x_{j+2} \geq x_{n}$, $y_{1} \geqslant \ldots \geqslant y_{j-1}>y_{j} \geqslant y_{j+1}>y_{j+2} \geqslant \ldots \geqslant y_{n}, y \in C$.

Let $\Lambda^{\prime \prime}$ be the orbit $W y \subset P$. It is easy to see that $\AA^{\prime} \supset \Lambda^{\prime} \sqcup \AA^{\prime \prime}$. Therefore, $2 n \geqslant\left|\Lambda^{\prime} \backslash H\right| \geqslant\left|\Lambda^{\prime} \backslash H\right|+\left|\AA^{\prime \prime} \backslash H\right|$. By Proposition 2.24, $\left|\Lambda^{\prime} \backslash H\right| \geqslant 2$, which implies the inequality $\left|\AA^{\prime \prime} \backslash H\right| \leqslant 2 n-2<2 n$. By Proposition 2.26, we have that either $\left|\left\{y_{1}, \ldots, y_{n}\right\}\right| \leqslant 2$ or $y \in \Delta$.

Suppose that $\left|\left\{y_{1}, \ldots, y_{n}\right\}\right| \leqslant 2$.

For the integers $k_{1}:=\left|\left\{y_{1}, \ldots, y_{j-1}\right\}\right|, k_{2}:=\left|\left\{y_{j}, y_{j+1}\right\}\right|, k_{3}:=\left|\left\{y_{j+2}, \ldots, y_{n}\right\}\right|$ we have $k_{1}, k_{3} \geqslant 0, k_{2} \geqslant 1,\left(k_{1}=0\right) \Rightarrow(j=1)$, and $\left(k_{3}=0\right) \Rightarrow(j=n-1)$. Furthermore, $n-1>1$, whence

$$
k_{1}+k_{3} \geqslant 1 ; \quad\left(k_{1}+k_{3}=1\right) \Rightarrow(j \in\{1, n-1\}) .
$$

Moreover, $\left(k_{1}+k_{3}\right)+k_{2}=\left|\left\{y_{1}, \ldots, y_{n}\right\}\right| \leqslant 2$ and $k_{2} \geqslant 1$. Therefore, $k_{1}+k_{3}=1$, $j \in\{1, n-1\}$, and $k_{2}=1, y_{j}=y_{j+1}, x_{j}-x_{j+1}=2$.

Suppose now that $y \in \Delta$.

By (2.9), $0 \notin\left\{x_{1}, \ldots, x_{n}\right\}$. It now follows from the relations $x=y+\alpha \in \Delta+\Delta$ and $x \in C$ that

$$
n \leqslant 4 ; \quad(n=4) \Rightarrow\left(x_{1}=x_{2}=1, x_{3}=x_{4}=-1\right) .
$$

Suppose $n=3$. Then for $r=2$,

$$
\operatorname{dim} H=r-1=1 .
$$

Furthermore,

$$
\begin{gathered}
\Lambda^{\prime \prime}=\Delta, \quad \stackrel{\circ}{\Lambda}^{\prime} \supset \Lambda^{\prime} \sqcup \Delta, \\
\left|\Lambda^{\prime}\right|+|\Delta| \leqslant\left|\Lambda^{\prime} \cap H\right|+|\Delta \cap H|+\left|\stackrel{\circ}{\Lambda}^{\prime} \backslash H\right| \leqslant\left|\Lambda^{\prime} \cap H\right|+|\Delta \cap H|+2 n, \\
3+n(n-1) \leqslant\left|\Lambda^{\prime} \cap H\right|+|\Delta \cap H|+2 n, \\
\left|\Lambda^{\prime} \cap H\right|+|\Delta \cap H| \geqslant n(n-3)+3=3,
\end{gathered}
$$

and, according to (2.10),

$$
\begin{aligned}
& \left|\Lambda^{\prime} \cap H\right|,|\Delta \cap H| \leqslant 2<3 \leqslant\left|\Lambda^{\prime} \cap H\right|+|\Delta \cap H|, \\
& \left|\Lambda^{\prime} \cap H\right|,|\Delta \cap H|>0, \quad \Lambda^{\prime} \cap H, \Delta \cap H \neq \varnothing .
\end{aligned}
$$

But, by (2.9), no root of $\Delta \subset \mathbb{R}^{n}$ is proportional to a vector from the orbit $\Lambda^{\prime}=W x \subset P$, contrary to (2.10).

Thus, the proposition is proved.

Corollary 2.28. Let $x \in P \cap C$ be a vector and let $\Lambda^{\prime}$ denote the orbit $W x \subset P$. If $x_{1}-x_{n}>2$, then $\left|\Lambda^{\prime} \backslash H\right|>2 n$ and $\left|\Lambda^{\prime} \backslash H\right| \geqslant 2$.

Proof. Follows from Propositions 2.24, 2.26, and 2.27.

Proposition 2.29. Let $x \in P \cap C$ be a vector, and let $\Lambda^{\prime}$ denote the orbit $W x \subset P$. If $x \notin \Delta, x_{1}-x_{n}>1,-\Lambda^{\prime}=\Lambda^{\prime}$, and $\left|\AA^{\prime} \backslash H\right| \leqslant 4 n$, then $n=4, x_{1}=x_{2}=1$, and $x_{3}=x_{4}=-1$.

Proof. We have $x_{n+1-i}=-x_{i}$ for any $i=1, \ldots, n$. Furthermore, since $n-1 \geqslant 2$, we have $x_{2} \geqslant x_{n-1}=-x_{2}, x_{2} \geqslant 0$.

We can have the following cases. 
Case 1. $x_{1}-x_{n}>2$ and $x_{2}>0$.

Case 2. $x_{1}-x_{n}>2$ and $x_{2}=0$.

Case 3. $x_{1}-x_{n}=2$.

First consider Case 1 .

We have $x_{1}=\frac{\left(x_{1}-x_{n}\right)}{2}>1$.

Set $\alpha:=\varepsilon_{1}-\varepsilon_{n} \in \Delta$. Because $\langle x \mid \alpha\rangle=x_{1}-x_{n}>2$ and $x \notin \Delta$, we have $y:=x-\alpha \in \stackrel{\circ}{\prime}^{\prime} \backslash\left(\Lambda^{\prime} \sqcup\{0\}\right)$. Let $\Lambda^{\prime \prime}$ denote the orbit $W y \subset P$. We have $\Lambda^{\prime \prime} \cap C=\{\widetilde{y}\}$ and $\widetilde{y} \in(P \cap C) \backslash\{0\}$. Moreover,

$$
\stackrel{\circ}{\Lambda}^{\prime} \supset \Lambda^{\prime} \sqcup \Lambda^{\prime \prime}, \quad 4 n \geqslant\left|\Upsilon^{\prime} \backslash H\right| \geqslant\left|\Lambda^{\prime} \backslash H\right|+\left|\Lambda^{\prime \prime} \backslash H\right|,
$$

and, by Proposition 2.24, $\left|\Lambda^{\prime} \backslash H\right|$ and $\left|\Lambda^{\prime \prime} \backslash H\right|<4 n$.

By Proposition 2.22, $\left|\left\{x_{1}, \ldots, x_{n}\right\}\right|,\left|\left\{\widetilde{y}_{1}, \ldots, \widetilde{y}_{n}\right\}\right|<4,\left|\left\{y_{1}, \ldots, y_{n}\right\}\right|<4$. Moreover, $\left\{x_{1}, \ldots, x_{n}\right\} \supset\left\{x_{1}, x_{2}, x_{n-1}, x_{n}\right\}=\left\{ \pm x_{1}, \pm x_{2}\right\},\left\{y_{1}, \ldots, y_{n}\right\}=\left\{y_{1}, x_{2}, \ldots, x_{n-1}, y_{n}\right\}$, $y_{1}=x_{1}-1>0, y_{n}=x_{n}+1=-x_{1}+1=-y_{1},\left\{y_{1}, \ldots, y_{n}\right\} \supset\left\{ \pm y_{1}, \pm x_{2}\right\}$. Thus, $\left|\left\{ \pm x_{1}, \pm x_{2}\right\}\right|<4$ and $\left|\left\{ \pm y_{1}, \pm x_{2}\right\}\right|<4$. On the other hand, $x_{1}, y_{1}, x_{2}>0$. This implies that $x_{2}=x_{1}$ and $x_{2}=y_{1}=x_{1}-1$.

Thus Case 1 leads to a contradiction.

Now consider Case 2

We have

$$
x_{n-1}=-x_{2}=0, \quad x_{2}=\ldots=x_{n-1}=0, \quad x=x_{1}\left(\varepsilon_{1}-\varepsilon_{n}\right) .
$$

Furthermore, $x_{1}=x_{1}-x_{2} \in \mathbb{Z}$, and also $x_{1}=\frac{\left(x_{1}-x_{n}\right)}{2}>1$, whence $x_{1} \geqslant 2$. Notice that $x_{1}-1 \geqslant 1>0>-x_{1}$ and, as a consequence,

$$
y:=\left(x_{1}-1\right) \varepsilon_{1}+\varepsilon_{2}-x_{1} \varepsilon_{n} \in\left(\stackrel{\circ}{\Lambda}^{\prime} \backslash \Lambda^{\prime}\right) \cap C \subset P .
$$

The orbits $\Lambda^{\prime \prime}:=W y \subset P$ and $-\Lambda^{\prime \prime} \subset P$ are distinct, since $y_{1}+y_{n}=\left(x_{1}-1\right)-x_{1} \neq 0$. Therefore $\AA^{\prime} \supset \Lambda^{\prime} \sqcup\left(-\Lambda^{\prime \prime}\right) \sqcup \AA^{\prime \prime}$ and $4 n \geqslant\left|\AA^{\prime} \backslash H\right| \geqslant\left|\Lambda^{\prime} \backslash H\right|+\left|\Lambda^{\prime \prime} \backslash H\right|+\left|\Lambda^{\prime \prime} \backslash H\right|$. $\quad$ Moreover,

1) $\left|\left\{x_{1}, \ldots, x_{n}\right\}\right|=\left|\left\{ \pm x_{1}, 0\right\}\right|=3$, and, according to Proposition 2.22, $\left|\Lambda^{\prime} \backslash H\right| \geqslant 2 n-2$;

2) $y_{1}-y_{n}=\left(x_{1}-1\right)+x_{1}=2 x_{1}-1 \geqslant 3$, and, by Corollary $2.28,\left|\Lambda^{\prime \prime} \backslash H\right|+\left|\Lambda^{\prime \prime} \backslash H\right|>$ $2 n+2$.

Hence,

$$
\left|\Lambda^{\prime} \backslash H\right|+\left|\Lambda^{\prime \prime} \backslash H\right|+\left|\stackrel{\Lambda}{\prime \prime}^{\prime \prime} \backslash H\right|>(2 n-2)+(2 n+2)=4 n .
$$

This is a contradiction.

Finally, consider Case 3 .

We have $x_{1}=\frac{\left(x_{1}-x_{n}\right)}{2}=1, x=\left(\varepsilon_{1}+\ldots+\varepsilon_{j}\right)-\left(\varepsilon_{n+1-j}+\ldots+\varepsilon_{n}\right), j \in \mathbb{N}, j \leqslant \frac{n}{2}$. By assumption, $x \notin \Delta$, whence $j \geqslant 2, n \geqslant 4$. If $n=4$, then $j=2, x_{1}=x_{2}=1$, and $x_{3}=x_{4}=-1$.

Suppose that $n>4$. Set $y:=\left(\varepsilon_{1}+\varepsilon_{2}\right)-\left(\varepsilon_{n-1}+\varepsilon_{n}\right) \in P \cap C$. It is easy to see that $\stackrel{\Lambda}{ }^{\prime} \supset(W y) \sqcup \Delta$. Hence, $4 n \geqslant\left|\AA^{\prime} \backslash H\right| \geqslant|(W y) \backslash H|+|\Delta \backslash H|$ and, according to Corollary 2.23. $|(W y) \backslash H| \leqslant 4 n-|\Delta \backslash H| \leqslant 4 n-(2 n-2)=2 n+2$. Furthermore, $n-4>0,\left|\left\{y_{1}, \ldots, y_{n}\right\}\right|=3$ and, also, $n-2>2, \max \{2, n-4\}<n-2$. This together with Proposition 2.22 implies that

$$
|(W y) \backslash H| \geqslant 5(n-2)=(2 n+2)+3(n-4)>2 n+2 .
$$

This is a contradiction.

The proposition is proved. 
Applying Propositions 2.26, 2.27 and 2.29 to the vector $\lambda \in(P \cap C) \backslash\{0\}$ and its orbit $\Lambda \subset P \backslash\{0\}$, we have Lemma 2.20 .

Now let us prove Lemma 2.21.

By assumption, $j \in\{3, \ldots, n-3\}, \lambda_{1}=\ldots=\lambda_{j}, \lambda_{j+1}=\ldots=\lambda_{n}$, and $\lambda_{j}-\lambda_{j+1}=1$. In particular, $\lambda_{n-1}=\lambda_{n}$, and therefore

$$
H=\left\{x \in\langle\Delta\rangle: x_{n-1}=x_{n}\right\}, \quad|\Lambda \backslash H|=\left(\begin{array}{l}
2 \\
1
\end{array}\right) \cdot\left(\begin{array}{c}
n-2 \\
j-1
\end{array}\right) .
$$

Moreover $2 \leqslant j-1 \leqslant(n-2)-2$, whence

$$
\left(\begin{array}{l}
n-2 \\
j-1
\end{array}\right) \geqslant\left(\begin{array}{c}
n-2 \\
2
\end{array}\right)=\frac{(n-2)(n-3)}{2} .
$$

Therefore,

$$
|\Lambda \backslash H| \geqslant(n-2)(n-3)>n(n-5)=n(r-4)=4 n+n(r-8) \geqslant 4 n .
$$

Thus Lemma 2.21 is proved.

2.4.2. Additional results. Here we prove Propositions 2.30 2.33

For arbitrary, pairwise distinct numbers $i_{1}, \ldots, i_{k} \in\{1, \ldots, n\}$, where $k=1, \ldots, n$, we set

$$
\lambda_{\left(i_{1}, \ldots, i_{k}\right)}:=\left(\varepsilon_{i_{1}}+\ldots+\varepsilon_{i_{k}}\right)-\frac{k}{n} \cdot\left(\varepsilon_{1}+\ldots+\varepsilon_{n}\right) \in P .
$$

Furthermore, for arbitrary pairwise distinct numbers $i, i_{1}, i_{2} \in\{1, \ldots, n\}$ we set

$$
\begin{aligned}
\lambda_{\left(i_{1}, i_{2}\right)}^{(i)} & :=\lambda_{\left(i_{1}\right)}+\left(\varepsilon_{i_{2}}-\varepsilon_{i}\right)=\lambda_{\left(i_{2}\right)}+\left(\varepsilon_{i_{1}}-\varepsilon_{i}\right) \\
& =\left(\varepsilon_{i_{1}}+\varepsilon_{i_{2}}-\varepsilon_{i}\right)-\frac{1}{n} \cdot\left(\varepsilon_{1}+\ldots+\varepsilon_{n}\right) \in P .
\end{aligned}
$$

Proposition 2.30. If $r>2$ and $\lambda=\varphi_{2}+\varphi_{r}$, then there are a subset $\Omega \subset \Lambda$ and $a$ hyperplane $H \subset\langle\Delta\rangle$ such that $\langle\Omega\rangle=H,(\Omega-\Omega) \cap \Delta=\varnothing$ and $2 \cdot|\AA \backslash H|>|\Delta \backslash H|+6$.

Proof. We have $n=r+1 \geqslant 4, \lambda=\lambda_{(1,2)}^{(n)}$, and $\AA=\Lambda \sqcup\left\{\lambda_{(1)}, \ldots, \lambda_{(n)}\right\}$.

Set $\Omega:=\left\{\lambda_{(i-1, i)}^{(i+1)}: i=2, \ldots, r\right\} \subset \Lambda$ and $H:=\langle\Omega\rangle \subset\langle\Delta\rangle$. Let us prove that the subset $\Omega \subset \Lambda$ and the subspace $H \subset\langle\Delta\rangle$ satisfy the desired requirements.

An arbitrary vector of the form $\left(\varepsilon_{j-1}+\varepsilon_{j}-\varepsilon_{j+1}\right)-\left(\varepsilon_{i-1}+\varepsilon_{i}-\varepsilon_{i+1}\right) \in P(2 \leqslant i<j \leqslant$ $r)$ has its $(i+1)$-st coordinate equal to 2 when $j \leqslant i+2$, and six nonzero coordinates when $j>i+2$. Hence, $(\Omega-\Omega) \cap \Delta=\varnothing$.

The subspace $H^{\prime}:=H^{\perp} \cap\langle\Delta\rangle \subset \mathbb{R}^{n}$ is given by the equations $x_{i-1}+x_{i}=x_{i+1}$, where $i=2, \ldots, r$, and $x_{1}+\ldots+x_{n}=0$. Moreover,

$$
\operatorname{dim} H \leqslant|\Omega|=r-1, \quad \operatorname{dim} H^{\prime}=r-\operatorname{dim} H \geqslant 1 .
$$

Let $x \in H^{\prime}$ be an arbitrary nonzero vector.

Let us show that $x_{1}, x_{2}, x_{3}, x_{4} \neq 0$. We have $x_{i-1}+x_{i}=x_{i+1}(i=2, \ldots, r)$ and $x_{1}+\ldots+x_{n}=0$, whence

$$
\begin{gathered}
\forall i=2, \ldots, r \quad x_{i-1} x_{i} \leqslant x_{i-1} x_{i}+x_{i}^{2}=x_{i} x_{i+1} ; \\
2 x_{3}+\sum_{i=4}^{n} x_{i}=\left(x_{1}+x_{2}+x_{3}\right)+\sum_{i=4}^{n} x_{i}=\sum_{i=1}^{n} x_{i}=0 \\
4 x_{3}^{2}+4 \cdot \sum_{i=4}^{n} x_{3} x_{i}+\sum_{i_{1}, i_{2}=4}^{n} x_{i_{1}} x_{i_{2}}=0 .
\end{gathered}
$$


Suppose that there is a pair $\left(i_{1}, i_{2}\right) \in\{3, \ldots, n\}^{2}$ such that $i_{1} \leqslant i_{2}$ and $x_{i_{1}} x_{i_{2}}<0$. Among all such pairs choose a pair $\left(i_{1}, i_{2}\right)$ with smallest $i_{1}+i_{2}$. We have $i_{1}<i_{2}$. Furthermore, if $i_{2} \geqslant 5$, then

$$
3 \leqslant i_{2}-2<i_{2}-1<n \quad \text { and } \quad i_{1}+\left(i_{2}-2\right)<i_{1}+\left(i_{2}-1\right)<i_{1}+i_{2}
$$

whence $x_{i_{1}} x_{i_{2}-2}, x_{i_{1}} x_{i_{2}-1} \geqslant 0, x_{i_{1}}\left(x_{i_{2}-2}+x_{i_{2}-1}\right) \geqslant 0$, and $x_{i_{1}} x_{i_{2}} \geqslant 0$, contrary to the assumption. Hence, $i_{2}<5,3 \leqslant 1<i_{2}<5$, and therefore $\left(i_{1}, i_{2}\right)=(3,4)$ and $x_{3} x_{4}<0$. By (2.11), $x_{1} x_{2} \leqslant x_{2} x_{3} \leqslant x_{3} x_{4}<0, x_{1}, x_{2}, x_{3}, x_{4} \neq 0$.

Suppose now that $x_{i_{1}} x_{i_{2}} \geqslant 0$ for all $i_{1}, i_{2} \in\{3, \ldots, n\}\left(i_{1} \leqslant i_{2}\right)$. Then, according to (2.12), $x_{3}^{2}=\ldots=x_{n}^{2}=0, x_{3}=\ldots=x_{n}=0, x_{2}=x_{4}-x_{3}=0, x_{1}=x_{3}-x_{2}=0$, $x_{1}=\ldots=x_{n}=0$, whereas $x \neq 0$. This is a contradiction.

We have thus established that $\operatorname{dim} H^{\prime} \geqslant 1$ and, moreover, $x_{1}, x_{2}, x_{3}, x_{4} \neq 0$ for any nonzero vector $x \in H^{\prime}$. As a consequence, $H^{\prime}=\mathbb{R} x \subset\langle\Delta\rangle\left(x \in\langle\Delta\rangle, x_{1}, x_{2}, x_{3}, x_{4} \neq 0\right)$, $\operatorname{dim} H^{\prime}=1, H=\left(H^{\prime}\right)^{\perp} \cap\langle\Delta\rangle=\{y \in\langle\Delta\rangle:(y, x)=0\} \subset \mathbb{R}^{n}, \operatorname{dim} H=r-1$.

It remains to show that $2 \cdot|\AA \backslash H|>|\Delta \backslash H|+6$.

For any $i=1,2,3,4$, we have $\left(\lambda_{(i)}, x\right)=\left(\varepsilon_{i}, x\right)=x_{i} \neq 0$, whence $\lambda_{(i)} \in \AA \backslash H$. Therefore, $|\AA \backslash H| \geqslant|\Lambda \backslash H|+4>|\Lambda \backslash H|+3$.

Let $I=\left\{i_{1}, i_{2}\right\} \subset\{1, \ldots, n\}$ be an arbitrary two-element subset. Let us prove that there is a number $i \in\{1, \ldots, n\} \backslash I$ such that $x_{i} \neq x_{i_{1}}+x_{i_{2}}$.

Suppose that $x_{i}=x_{i_{1}}+x_{i_{2}}$ for any $i \in\{1, \ldots, n\} \backslash I$. Then

$$
0=\sum_{i=1}^{n} x_{i}=\left(x_{i_{1}}+x_{i_{2}}\right)+(n-2)\left(x_{i_{1}}+x_{i_{2}}\right)=(n-1)\left(x_{i_{1}}+x_{i_{2}}\right), \quad x_{i_{1}}+x_{i_{2}}=0 .
$$

Thus, all numbers $x_{i} \in \mathbb{R}$ (where $i \in\{1, \ldots, n\}$ and $i \neq i_{1}, i_{2}$ ) are zero, which is impossible since $x_{1}, x_{2}, x_{3}, x_{4} \neq 0$.

According to the above, for all distinct numbers $i_{1}, i_{2} \in\{1, \ldots, n\}$ there is a number $i \in\{1, \ldots, n\} \backslash\left\{i_{1}, i_{2}\right\}$ such that $x_{i} \neq x_{i_{1}}+x_{i_{2}}$ (and therefore $\left(\varepsilon_{i_{1}}+\varepsilon_{i_{2}}-\varepsilon_{i}, x\right) \neq 0$, $\left.\left(\lambda_{\left(i_{1}, i_{2}\right)}^{(i)}, x\right) \neq 0, \lambda_{\left(i_{1}, i_{2}\right)}^{(i)} \in \Lambda \backslash H\right)$. Hence,

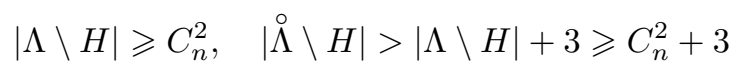

and, therefore, $2 \cdot|\AA \backslash H|>2 \cdot C_{n}^{2}+6=|\Delta|+6 \geqslant|\Delta \backslash H|+6$.

Proposition 2.31. If $r=3$ and $\lambda=\varphi_{1}+\varphi_{2}+\varphi_{3}$, then

1) $2 \lambda \notin \Delta \cup(\Delta+\Delta)$;

2) there are a subset $\Omega \subset \Lambda$ and a hyperplane $H \subset\langle\Delta\rangle$ such that $\langle\Omega\rangle=H,(\Omega+\Omega) \cap \Delta=$ $(\Omega-\Omega) \cap \Delta=\varnothing$, and $|\AA \backslash H|>|\Delta \backslash H|+6$.

Proof. We have

$$
\lambda=\frac{1}{2} \cdot\left(3\left(\varepsilon_{1}-\varepsilon_{4}\right)+\left(\varepsilon_{2}-\varepsilon_{3}\right)\right), \quad 2 \lambda=3\left(\varepsilon_{1}-\varepsilon_{4}\right)+\left(\varepsilon_{2}-\varepsilon_{3}\right) \notin \Delta \cup(\Delta+\Delta),
$$

$|\Lambda|=24$ and $|\Delta|=12$.

Set

$$
\Omega:=\left\{\frac{1}{2} \cdot\left(3\left(\varepsilon_{1}-\varepsilon_{4}\right)+\left(\varepsilon_{2}-\varepsilon_{3}\right)\right), \frac{1}{2} \cdot\left(3\left(\varepsilon_{1}-\varepsilon_{2}\right)+\left(\varepsilon_{4}-\varepsilon_{3}\right)\right)\right\} \subset \Lambda .
$$

As is easily seen,

$$
(\Omega+\Omega) \cap \Delta=(\Omega-\Omega) \cap \Delta=\varnothing \quad \text { and } \quad H:=\langle\Omega\rangle=\left\{x \in\langle\Delta\rangle: x_{1}+3 x_{3}=0\right\} \subset\langle\Delta\rangle .
$$

Furthermore,

$$
\Lambda \cap H=\Omega \sqcup(-\Omega) \subset \Lambda, \quad|\Lambda \cap H|=4, \quad|\Lambda \backslash H|=20>|\Delta|+6 \geqslant|\Delta \backslash H|+6 .
$$


Proposition 2.32. If $r=6$ and $\lambda=\varphi_{3}$, then there are a subset $\Omega \subset \Lambda$ and a hyperplane $H \subset\langle\Delta\rangle$ such that $\langle\Omega\rangle=H,(\Omega-\Omega) \cap \Delta=\varnothing$, and $2 \cdot|\AA \backslash \Lambda|>|\Delta \backslash H|+6$.

Proof. By assumption, $\lambda=\lambda_{(1,2,3)}$ and $|\Lambda|=35$.

Set

$$
\Omega:=\left\{\lambda_{(1,2,5)}, \lambda_{(3,4,5)}, \lambda_{(1,4,6)}, \lambda_{(2,3,6)}, \lambda_{(5,6,7)}\right\} \subset \Lambda
$$

We have

$$
(\Omega-\Omega) \cap \Delta=\varnothing \quad \text { and } \quad H:=\langle\Omega\rangle=\left\{x \in\langle\Delta\rangle: x_{1}+x_{3}=x_{2}+x_{4}\right\} \subset\langle\Delta\rangle .
$$

Hence, $|\Delta \backslash H|=n^{2}-\left(3^{2}+2^{2}+2^{2}\right)=32$. Moreover,

$$
\Lambda \cap H=\left\{\lambda_{\left(i_{1}, i_{2}, i_{3}\right)} \in P: i_{1} \in\{1,3\}, i_{2} \in\{2,4\}, i_{3} \in\{5,6,7\}\right\} \sqcup\left\{\lambda_{(5,6,7)}\right\} \subset \Lambda,
$$

$|\Lambda \cap H|=13,|\Lambda \backslash H|=22,2 \cdot|\Lambda \backslash H|=44>|\Delta \backslash H|+6$.

Proposition 2.33. If $r=7$ and $\lambda=\varphi_{3}$, then there are a subset $\Omega \subset \Lambda$ and a hyperplane $H \subset\langle\Delta\rangle$ such that $\langle\Omega\rangle=H,(\Omega-\Omega) \cap \Delta=\varnothing$ and $2 \cdot|\AA \backslash H|>|\Delta \backslash H|+6$.

Proof. We have $\lambda=\lambda_{(1,2,3)}$ and $|\Lambda|=|\Delta|=56$.

Set

$$
\Omega:=\left\{\lambda_{(1,2,7)}, \lambda_{(3,4,7)}, \lambda_{(5,6,7)}, \lambda_{(4,5,8)}, \lambda_{(1,6,8)}, \lambda_{(2,3,8)}\right\} \subset \Lambda .
$$

As is easily seen,

$$
(\Omega-\Omega) \cap \Delta=\varnothing \quad \text { and } \quad H:=\langle\Omega\rangle=\left\{x \in\langle\Delta\rangle: x_{1}+x_{3}+x_{5}=x_{2}+x_{4}+x_{6}\right\} \subset\langle\Delta\rangle .
$$

Furthermore,

$$
\Lambda \cap H=\left\{\lambda_{\left(i_{1}, i_{2}, i_{3}\right)} \in P: i_{1} \in\{1,3,5\}, i_{2} \in\{2,4,6\}, i_{3} \in\{7,8\}\right\} \subset \Lambda,
$$

whence $|\Lambda \cap H|=18,|\Lambda \backslash H|=38$, and $2 \cdot|\Lambda \backslash H|=76>|\Delta|+6 \geqslant|\Delta \backslash H|+6$.

\section{$\S 3$. Proofs of the MAIn Results}

This section is devoted to proving Theorem 1.1

We return to the notation and the assumptions from $₫ 1$

Set $\delta:=1 \in \mathbb{R}$ if the representation $R$ of the algebra $\mathfrak{g}_{\mathbb{C}}$ is orthogonal, and $\delta:=2 \in \mathbb{R}$ otherwise.

Fix a maximal commutative subalgebra $\mathfrak{t}$ of $\mathfrak{g}$ and a Cartan subalgebra $\mathfrak{t}_{\mathbb{C}}:=\mathfrak{t} \otimes \mathbb{C}$ of $\mathfrak{g}_{\mathbb{C}}$. As a result, we have a root system $\Delta \subset \mathfrak{t}_{\mathbb{C}}^{*}$ and its Weyl group $W \subset \mathbf{G L}\left(\mathfrak{t}_{\mathbb{C}}^{*}\right)$. We have $\mathfrak{g} \cong \mathfrak{s u}_{r+1}, r>1$, and therefore $\Delta \subset \mathfrak{t}_{\mathbb{C}}^{*}$ is an indecomposable root system of type $A_{r}$. Moreover,

$$
\langle\Delta\rangle=\left\{\lambda \in \mathfrak{t}_{\mathbb{C}}^{*}: \lambda(\mathfrak{t}) \subset i \mathbb{R}\right\} \subset \mathfrak{t}_{\mathbb{C}}^{*}=\langle\Delta\rangle \oplus i\langle\Delta\rangle .
$$

Fix a simple root system $\Pi \subset \Delta$ and the corresponding Weyl chamber $C \subset\langle\Delta\rangle \subset \mathfrak{t}_{\mathbb{C}}^{*}$.

Let $P$ and $Q$ denote the lattices $\{\lambda \in\langle\Delta\rangle:\langle\lambda \mid \alpha\rangle \in \mathbb{Z} \forall \alpha \in \Delta\} \subset\langle\Delta\rangle$ and $\langle\Delta\rangle_{\mathbb{Z}} \subset$ $\langle\Delta\rangle$, respectively. We have $Q \subset P$. Let $\lambda \in(P \cap C) \backslash\{0\}$ be the highest weight representation $R$ of $\mathfrak{g}_{\mathbb{C}}$ relative to the simple root system $\Pi \subset \Delta \subset \mathfrak{t}_{\mathbb{C}}^{*}$.

Set $\Lambda:=W \lambda \subset P, \stackrel{\AA}{\Lambda}:=\operatorname{conv}(\Lambda) \cap(\Lambda+Q) \subset P$, and $n:=r+1 \in \mathbb{N}$. It is easy to see that the set of weights of the representation $R$ of $\mathfrak{g}_{\mathbb{C}}$ coincides with the subset $\Lambda \subset P$.

To prove Theorem 1.1 assume the opposite: suppose that $V / G$ is a smooth manifold and the linear algebra $R\left(\mathfrak{g}_{\mathbb{C}}\right)$ is not isomorphic to any of the linear algebras $\operatorname{ad}\left(\mathfrak{g}_{\mathbb{C}}\right)$, 
$\varphi_{1}\left(A_{r}\right), \varphi_{2}\left(A_{r}\right),\left(2 \varphi_{1}\right)\left(A_{r}\right)(r>1),\left(2 \varphi_{2}\right)\left(A_{3}\right), \varphi_{3}\left(A_{5}\right)$ and $\varphi_{4}\left(A_{7}\right)$. The latter condition means that

$$
\begin{aligned}
\lambda \notin \Delta \cup\left\{\varphi_{1}, \varphi_{2}, \varphi_{r-1}, \varphi_{r}, 2 \varphi_{1}, 2 \varphi_{r}\right\} ; & \\
& (r=3) \Rightarrow\left(\lambda \neq 2 \varphi_{2}\right) ; \\
& (r=5) \Rightarrow\left(\lambda \neq \varphi_{3}\right) ; \\
& (r=7) \Rightarrow\left(\lambda \neq \varphi_{4}\right) .
\end{aligned}
$$

Moreover, since the linear algebras $\varphi_{j}\left(A_{r}\right)$ and $\varphi_{n-j}\left(A_{r}\right)(j=1, \ldots, r)$, and also the linear algebras $\left(\varphi_{1}+\varphi_{r-1}\right)\left(A_{r}\right)$ and $\left(\varphi_{2}+\varphi_{r}\right)\left(A_{r}\right)$, are isomorphic, we may (and will) assume without loss of generality that

$$
\left(\lambda=\varphi_{j}, j=1, \ldots, r\right) \Rightarrow\left(j \leqslant \frac{n}{2}\right) ; \quad \lambda \neq \varphi_{1}+\varphi_{r-1} .
$$

Set $\Pi_{\lambda}:=\{\alpha \in \Pi:\langle\lambda \mid \alpha\rangle \neq 0\} \subset \Pi$. Also, let $\mathcal{P}$ denote the family of all indecomposable simple root systems $\Pi^{\prime} \subset \Pi \subset \mathfrak{t}_{\mathbb{C}}^{*}$ of order $r-2$.

Assertion 3.1. Suppose that $r>2$. Then the simple root system $\Pi$ coincides with the union of all of its subsets $\Pi^{\prime} \in \mathcal{P}$.

Proof. See 3.1 of [5, §3].

Lemma 3.2. Let $\Pi^{\prime} \in \mathcal{P}$ be a simple root system such that $(r>2) \Rightarrow\left(\Pi_{\lambda} \cap \Pi^{\prime} \neq \varnothing\right)$. If $\lambda \neq \varphi_{2}+\varphi_{r}$, then at least one of the following conditions holds:

$$
\begin{array}{ll}
\lambda \in\left\{\varphi_{3}, \ldots, \varphi_{r-2}\right\} ; & \left(\Pi^{\prime}=(1, \ldots, r-2) \subset \Pi\right) \Rightarrow(r<8) ; \\
r>2 ; & \Pi_{\lambda} \cap \Pi^{\prime}=\{\alpha\} \subset \Pi, \quad \alpha \in \partial \Pi^{\prime} \subset \Pi, \quad\langle\lambda \mid \alpha\rangle=1 .
\end{array}
$$

Proof. It is clear that $H:=\left\langle\{\lambda\} \cup \Pi^{\prime}\right\rangle \subset\langle\Delta\rangle \subset \mathfrak{t}_{\mathbb{C}}^{*}$ is an $(r-1)$-dimensional (real) subspace and, therefore, the intersection of the kernels of all linear functions on that subspace is of the form $\mathbb{C} \xi \subset \mathfrak{t}_{\mathbb{C}}, \xi \in \mathfrak{t} \backslash\{0\}$.

We have $\Pi \cong A_{r}$ and $\Pi^{\prime} \cong A_{r-2}$. As a consequence,

$$
|\Delta|=r(r+1) \quad \text { and } \quad\left|\Delta \cap\left\langle\Pi^{\prime}\right\rangle\right|=(r-2)(r-1) .
$$

Hence,

$$
\begin{aligned}
& |\Delta \cap H| \geqslant\left|\Delta \cap\left\langle\Pi^{\prime}\right\rangle\right|=(r-2)(r-1), \\
& |\Delta \backslash H| \leqslant r(r+1)-(r-2)(r-1)=4 r-2 .
\end{aligned}
$$

Suppose

$$
\exists v \in V \quad \xi \in \mathfrak{g}_{v}, \operatorname{rk} \mathfrak{g}_{v}=1
$$

By Lemma 2.12,

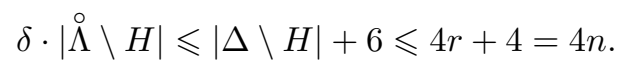

Moreover, $(\delta=1) \Rightarrow(-\Lambda=\Lambda)$. Thus

$$
|\AA \backslash H| \leqslant 4 n ; \quad(-\Lambda=\Lambda) \vee(|\AA \backslash H| \leqslant 2 n) .
$$

Furthermore, according to (3.2), $\lambda \notin\left\{\varphi_{1}+\varphi_{r-1}, \varphi_{2}+\varphi_{r}\right\}$. Now, using Lemmas 2.20 and 2.21, and the relations (3.1), we have (3.3).

If (3.5) does not hold, then, by Lemma 3.4 of [5, §3], we have the relations (3.4).

Corollary 3.3. Let $\Pi^{\prime} \in \mathcal{P}$ be a simple root system which does not satisfy (3.3). If $\lambda \neq \varphi_{2}+\varphi_{r}$, then

$$
\begin{gathered}
\left|\Pi_{\lambda} \cap \Pi^{\prime}\right| \leqslant 1, \quad \Pi_{\lambda} \cap\left(\Pi^{\prime} \backslash\left(\partial \Pi^{\prime}\right)\right)=\varnothing, \quad \forall \alpha \in \Pi_{\lambda} \cap \Pi^{\prime} \quad\langle\lambda \mid \alpha\rangle=1 ; \\
\left((r>2) \Rightarrow\left(\Pi_{\lambda} \cap \Pi^{\prime} \neq \varnothing\right)\right) \Rightarrow r>2 .
\end{gathered}
$$


Corollary 3.4. Let $\Pi^{\prime} \in \mathcal{P}$ be a simple root system which does not satisfy (3.3). If $\lambda \neq \varphi_{2}+\varphi_{r}$, then $r>2$ and (3.6) holds.

Suppose that $\lambda=\varphi_{2}+\varphi_{r}$.

By (3.1), $\lambda \neq 2 \varphi_{r}$, whence $r>2$. Furthermore, the representation $R$ of $\mathfrak{g}_{\mathbb{C}}$ is not self-adjoint, and therefore $\delta=2$. Now, using Proposition 2.30 and Corollary 2.13, we obtain a contradiction with the fact that $V / G$ is a smooth manifold.

Hence, $\lambda \neq \varphi_{2}+\varphi_{r}$. By (3.2), $\lambda \notin\left\{\varphi_{1}+\varphi_{r-1}, \varphi_{2}+\varphi_{r}\right\}$.

Suppose $\lambda \notin\left\{\varphi_{3}, \ldots, \varphi_{r-2}\right\}$.

None of the simple root systems $\Pi^{\prime} \in \mathcal{P}$ satisfies (3.3). Moreover, as is easily seen, $\mathcal{P} \neq \varnothing$. Corollary 3.4 implies that $r>2$ and that $(3.6)$ holds for any simple root system $\Pi^{\prime} \in \mathcal{P}$. Therefore,

1) for any $\alpha \in \Pi_{\lambda}$, we have $\langle\lambda \mid \alpha\rangle=1$ (see Assertion 3.1);

2 ) in the Dynkin diagram of the simple root system $\Pi$, the path between the vertices corresponding to two distinct roots from the subset $\Pi_{\lambda} \subset \Pi$ has at least $r-2$ edges. Hence, either $\lambda \in\left\{\varphi_{1}, \ldots, \varphi_{r}\right\} \cup\left\{\varphi_{1}+\varphi_{r}, \varphi_{1}+\varphi_{r-1}, \varphi_{2}+\varphi_{r}\right\}$ or $r=3$ and $\lambda=\varphi_{1}+\varphi_{2}+$ $\varphi_{3}$. At the same time, $\lambda \notin\left\{\varphi_{3}, \ldots, \varphi_{r-2}\right\} \cup\left\{\varphi_{1}+\varphi_{r-1}, \varphi_{2}+\varphi_{r}\right\}$. By (3.1), $r=3$ and $\lambda=\varphi_{1}+\varphi_{2}+\varphi_{3}$. The representation $R$ of $\mathfrak{g}_{\mathbb{C}}$ is orthogonal, and therefore $\delta=1$. By Proposition 2.31 and Corollary 2.13, the quotient $V / G$ is not a smooth manifold. On the other hand, $V / G$ is a smooth manifold. This is a contradiction.

Thus, we have established that $\lambda \in\left\{\varphi_{3}, \ldots, \varphi_{r-2}\right\}$.

We have $\lambda=\varphi_{j}, j \in \mathbb{N}, 3 \leqslant j \leqslant r-2$, whence $r \geqslant 5$. By (3.2), $j \leqslant \frac{n}{2}$.

If $r=5$, then $j=3, \lambda=\varphi_{3}$, contrary to (3.1).

Thus, $r \geqslant 6,3 \leqslant j \leqslant \frac{n}{2}$, and $\lambda=\varphi_{j}$. Moreover, by (3.1), $(r=7) \Rightarrow\left(\lambda \neq \varphi_{4}\right)$.

Case 1. $r=6$ and $\lambda=\varphi_{3}$.

The representation $R$ of $\mathfrak{g}_{\mathbb{C}}$ is not self-adjoint. Therefore $\delta=2$. Applying Proposition 2.32 and Corollary 2.13, we obtain a contradiction with the fact that $V / G$ is a smooth manifold.

Case 2. $r=7$ and $\lambda=\varphi_{3}$.

The representation $R$ of $\mathfrak{g}_{\mathbb{C}}$ is not self-adjoint. Hence, $\delta=2$. Using Proposition 2.33 and Corollary 2.13, we obtain a contradiction with the fact that $V / G$ is a smooth manifold.

Case 3. $r \geqslant 8$.

The simple root system $\Pi^{\prime}:=(1, \ldots, r-2) \subset \Pi$, belonging to the family $\mathcal{P}$, does not satisfy (3.3) and, by Corollary [3.4, satisfies (3.6). In particular, $\Pi_{\lambda} \cap\left(\Pi^{\prime} \backslash\left(\partial \Pi^{\prime}\right)\right)=\varnothing$. Moreover, $\Pi^{\prime} \cong A_{r-2}$ and $r-2 \geqslant 6$, whence $\partial \Pi^{\prime}=(1,2, r-3, r-2) \subset \Pi$ and $\Pi^{\prime} \backslash\left(\partial \Pi^{\prime}\right)=$ $(3, \ldots, r-4) \subset \Pi$. Furthermore,

$$
j \leqslant \frac{n}{2}=n-\frac{n}{2}<n-\frac{r}{2} \leqslant n-4=r-3, \quad 3 \leqslant j \leqslant r-4,
$$

and also $\Pi_{\lambda}=(j) \subset \Pi$. Hence, $\Pi_{\lambda} \cap\left(\Pi^{\prime} \backslash\left(\partial \Pi^{\prime}\right)\right)=(j) \neq \varnothing$. We have obtained a contradiction. Thus we have a complete ("contrapositive") proof of Theorem 1.1

\section{$\S$ 4. Particular cases}

In this section, we proveTheorem 1.2

As before, we shall use the notation and the assumptions from $\$ 1$

Set $n:=r+1 \in \mathbb{N}$.

Suppose the linear Lie group $G \subset \mathbf{G L}(V)$ is connected and the linear algebra $R\left(\mathfrak{g}_{\mathbb{C}}\right)$ is isomorphic to one of the following linear algebras: 

1) $\operatorname{ad}\left(\mathfrak{g}_{\mathbb{C}}\right)$;
2) $\varphi_{1}\left(A_{r}\right)(r>1)$
5) $\varphi_{2}\left(A_{3}\right) \cong \varphi_{1}\left(D_{3}\right)$;
3) $\left(2 \varphi_{1}\right)\left(A_{r}\right)(r>1)$;
6) $\left(2 \varphi_{2}\right)\left(A_{3}\right) \cong\left(2 \varphi_{1}\right)\left(D_{3}\right)$;
4) $\varphi_{2}\left(A_{r}\right)(r>3)$
7) $\varphi_{3}\left(A_{5}\right)$;
8) $\varphi_{4}\left(A_{7}\right)$.

First, consider cases 10, 20, (5), 6) and 8).

The following representations of the complex simple Lie groups are polar (see [, §3]):

- the adjoint representation of an arbitrary complex simple Lie group;

- the representation $R_{\varphi_{1}}+R_{\varphi_{1}}^{\prime}$ of the complex simple Lie group $\mathbf{S L}_{n}(\mathbb{C})$;

- the representation $R_{\varphi_{1}}$ and $R_{2 \varphi_{1}}$ of the complex simple Lie group $\mathbf{S O}_{6}(\mathbb{C})$;

- the representation $R_{\varphi_{4}}$ of the complex simple Lie group $\mathbf{S L}_{8}(\mathbb{C})$.

Hence, in each of the cases (1), 2), (5), 6), and 8) the linear Lie group $G \subset \mathbf{G L}(V)$ is polar and, by Lemma 2.16, the quotient $V / G$ is homeomorphic to a closed half-space (in particular, it is not a manifold).

Now we consider case 7).

Suppose that the linear group $G \subset \mathbf{G L}(V)$ is connected and the linear algebra $R\left(\mathfrak{g}_{\mathbb{C}}\right)$ is isomorphic to the linear algebra $\varphi_{3}\left(A_{5}\right)$.

Consider an $n$-dimensional Hermitian space $\mathbb{E}$. It admits a direct sum decomposition into two orthogonal three-dimensional subspaces $\mathbb{E}_{+}$and $\mathbb{E}_{-}$.

It is clear that the tautological representation $G: V$ is isomorphic to the natural action $\mathbf{S U}(\mathbb{E}): \mathbb{E}^{\wedge 3}$. Without loss of generality we may assume that $G=\mathbf{S U}(\mathbb{E}), V=\mathbb{E}^{\wedge 3}$, and the tautological representation $G: V$ coincides with the natural action $\mathbf{S U}(\mathbb{E}): \mathbb{E}^{\wedge 3}$.

As is easily seen, the space $V$ decomposes into a direct sum of the subspaces $V_{j}:=$ $\mathbb{E}_{+}^{\wedge(3-j)} \wedge \mathbb{E}_{-}^{\wedge j}(j=0,1,2,3)$, and $V_{0}=\mathbb{C} v, v \in V_{0} \backslash\{0\}$. Furthermore, $G_{v}=\mathbf{S U}\left(\mathbb{E}_{+}\right) \times$ $\mathbf{S U}\left(\mathbb{E}_{-}\right) \subset G$, where $\mathbf{S U}\left(\mathbb{E}_{ \pm}\right):=\left\{g \in G: \mathbb{E}^{g} \supset \mathbb{E}_{\mp}\right\} \subset G$. As a result, $G_{v} V_{j}=V_{j}$ $(j=0,1,2,3), \mathfrak{g} v=(i \mathbb{R} v) \oplus V_{1}$, and $V=(\mathfrak{g} v) \oplus(\mathbb{R} v) \oplus V_{2} \oplus V_{3}, V_{3} \subset V^{G_{v}}, \operatorname{dim}_{\mathbb{C}} V_{3}=1$. Therefore, the representation $G_{v}: N_{v}$ is isomorphic to the direct sum of the trivial action $G_{v}: \mathbb{R}^{3}$ and the representation $G_{v}: V_{2}$. The latter is, in turn, isomorphic to the natural action $\left(\mathbf{S U}\left(\mathbb{E}_{+}\right) \times \mathbf{S U}\left(\mathbb{E}_{-}\right)\right):\left(\mathbb{E}_{+} \otimes \mathbb{E}_{-}^{\wedge 2}\right)$ and, equivalently, to the natural action $\left(\mathbf{S U}\left(\mathbb{E}_{+}\right) \times \mathbf{S U}\left(\mathbb{E}_{-}\right)\right):\left(\mathbb{E}_{+} \otimes \mathbb{E}_{-}^{*}\right)$. Now, setting $d:=m:=3 \in \mathbb{N}$ and applying Assertion 2.10 to the representation $G_{v}: N_{v}$, we have that the quotient of that representation is not a manifold. By Lemma 2.1, neither is the quotient $V / G$.

Now consider the remaining cases (3) and (4).

Henceforth, we shall assume that the linear group $G \subset \mathbf{G L}(V)$ is connected, and the linear algebra $R\left(\mathfrak{g}_{\mathbb{C}}\right)$ is isomorphic to one of the linear algebras $\left(2 \varphi_{1}\right)\left(A_{r}\right)(r>1)$ and $\varphi_{2}\left(A_{r}\right)$ $(r>3)$.

Set $\delta:=1 \in \mathbb{R}$ (respectively, $\delta:=-1 \in \mathbb{R}$ ) if the linear algebra $R\left(\mathfrak{g}_{\mathbb{C}}\right)$ is isomorphic to the linear algebra $\left(2 \varphi_{1}\right)\left(A_{r}\right)$ (respectively, the linear algebra $\left.\varphi_{2}\left(A_{r}\right)\right)$.

Suppose $\delta=-1$ and $n \in 2 \mathbb{Z}+1$.

The representation $R_{\varphi_{2}}+R_{\varphi_{2}}^{\prime}$ of the complex simple Lie group $\mathbf{S L}_{n}(\mathbb{C})$ is polar (see $\underline{6}$, $\S 3])$. By Lemma 2.16, the quotient $V / G$ is homeomorphic to a closed half-space and, therefore, is not a manifold.

Henceforth we shall assume that $(\delta=1) \vee(n \in 2 \mathbb{Z})$.

Consider an $n$-dimensional Hermitian space $\mathbb{E}$ with scalar multiplication $f_{0}(\cdot, \cdot)$, the complex space $B$ of all bilinear forms on $\mathbb{E}$ and the subspace $B_{\delta} \subset B$ of all forms $f \in B$ such that $f(x, y)=\delta \cdot f(y, x)(x, y \in \mathbb{E})$.

The tautological representation $G: V$ is isomorphic to the representation

$$
\begin{gathered}
\mathbf{S U}(\mathbb{E}): B_{\delta}, \quad(g f)(x, y):=f\left(g^{-1} x, g^{-1} y\right) \in \mathbb{C} \\
\left(g \in \mathbf{S U}(\mathbb{E}), \quad f \in B_{\delta}, \quad x, y \in \mathbb{E}\right) .
\end{gathered}
$$


There is a form $f_{1} \in B_{\delta}$ such that the (fixed) orthonormal basis of the Hermitian space $\mathbb{E}$ is orthonormal when $\delta=1$ and symplectic when $\delta=-1$. Moreover, there is clearly an antilinear operator $F: \mathbb{E} \rightarrow \mathbb{E}$ such that $F^{2}=\delta E \in \mathbf{U}(\mathbb{E})$ and $f_{1}(x, y)=$ $f_{0}(F x, y)(x, y \in \mathbb{E})$.

Let $\theta, \theta_{0}$, and $\theta_{1}$ be involutive automorphisms of the real Lie group $\mathbf{G L}_{\mathbb{C}}(\mathbb{E})$, uniquely determined by the relations

$$
\theta(g) F=F g \quad \text { and } \quad f_{i}\left(\theta_{i}(g) x, g y\right)=f_{i}(x, y) \quad\left(i=0,1, g \in \mathbf{G L}_{\mathbb{C}}(\mathbb{E}), x, y \in \mathbb{E}\right) .
$$

For arbitrary $g \in \mathbf{G L}_{\mathbb{C}}(\mathbb{E})$ and $x, y \in \mathbb{E}$, we have

$$
f_{0}\left(F \theta_{1}(g) x, g y\right)=f_{1}\left(\theta_{1}(g) x, g y\right)=f_{1}(x, y)=f_{0}(F x, y)=f_{0}\left(\theta_{0}(g) F x, g y\right),
$$

whence $F \theta_{1}(g)=\theta_{0}(g) F$. Therefore, $\theta_{0} \equiv \theta \circ \theta_{1}$ and $\theta_{0} \circ \theta_{1} \equiv \theta_{1} \circ \theta_{0} \equiv \theta$. The real Lie algebra Lie $\left(\mathbf{G L}_{\mathbb{C}}(\mathbb{E})\right)=\mathfrak{g l}_{\mathbb{C}}(\mathbb{E})$ has commuting involutive automorphisms $\sigma_{i}:=d \theta_{i}$ $(i=0,1)$. It is easy to see that $f_{i}\left(\sigma_{i}(\xi) x, y\right)+f_{i}(x, \xi y)=0\left(i=0,1, \xi \in \mathfrak{g l}_{\mathbb{C}}(\mathbb{E})\right.$, $x, y \in \mathbb{E}$ ). Hence, the automorphism $\sigma_{0}$ (respectively, $\left.\sigma_{1}\right)$ of the real Lie algebra $\mathfrak{g l}_{\mathbb{C}}(\mathbb{E})$ is antilinear (respectively, linear) over $\mathbb{C}$, and the subspace $\left(\mathfrak{g l}_{\mathbb{C}}(\mathbb{E})\right)^{-\sigma_{1}} \subset \mathfrak{g l}_{\mathbb{C}}(\mathbb{E})$ contains the subspace $\mathbb{C} E \subset \mathfrak{g l}_{\mathbb{C}}(\mathbb{E})$.

It is clear that $\left(\mathbf{G L}_{\mathbb{C}}(\mathbb{E})\right)^{\theta_{0}}=\mathbf{U}(\mathbb{E}) \subset \mathbf{G L}_{\mathbb{C}}(\mathbb{E})$. Furthermore, the map

$$
S: \mathfrak{g l}_{\mathbb{C}}(\mathbb{E}) \rightarrow B, \quad(S(\xi))(x, y):=f_{1}(x, \xi y) \in \mathbb{C} \quad\left(\xi \in \mathfrak{g l}_{\mathbb{C}}(\mathbb{E}), x, y \in \mathbb{E}\right)
$$

is an isomorphism of complex linear spaces. Moreover, for all $g \in \mathbf{S U}(\mathbb{E}), \xi \in \mathfrak{g l}_{\mathbb{C}}(\mathbb{E})$, and $x, y \in \mathbb{E}$ we have

$$
\begin{aligned}
(S(\xi))\left(g^{-1} x, g^{-1} y\right) & =f_{1}\left(g^{-1} x, \xi g^{-1} y\right)=f_{1}\left(x, \theta_{1}(g) \xi g^{-1} y\right) \\
& =\left(S\left(\theta_{1}(g) \xi g^{-1}\right)\right)(x, y) \\
\delta \cdot(S(\xi))(y, x) & =\delta \cdot f_{1}(y, \xi x)=f_{1}(\xi x, y) \\
& =-f_{1}\left(x, \sigma_{1}(\xi) y\right)=-\left(S\left(\sigma_{1}(\xi)\right)\right)(x, y) .
\end{aligned}
$$

This implies that

$$
S^{-1}\left(B_{\delta}\right)=\left(\mathfrak{g l}_{\mathbb{C}}(\mathbb{E})\right)^{-\sigma_{1}} \subset \mathfrak{g l}_{\mathbb{C}}(\mathbb{E}),
$$

and the representation (4.1) is isomorphic to the representation

$$
\mathbf{S U}(\mathbb{E}):\left(\mathfrak{g l}_{\mathbb{C}}(\mathbb{E})\right)^{-\sigma_{1}}, \quad g: \xi \rightarrow \theta_{1}(g) \xi g^{-1} .
$$

As we already mentioned, the tautological representation $G: V$ is isomorphic to the representation (4.1), and therefore to the representation (4.2). Without loss of generality we may assume that $G=\mathbf{S U}(\mathbb{E}) \subset \mathbf{G L}_{\mathbb{C}}(\mathbb{E}), V=\left(\mathfrak{g l}_{\mathbb{C}}(\mathbb{E})\right)^{-\sigma_{1}} \subset \mathfrak{g l}_{\mathbb{C}}(\mathbb{E})$, and the tautological representation $G: V$ coincides with the representation (4.2).

The commuting automorphisms $\theta_{0}$ and $\theta_{1}$ of the Lie group $\mathbf{G L}_{\mathbb{C}}(\mathbb{E})$ preserve the commutant $\mathbf{S L}_{\mathbb{C}}(\mathbb{E})$ and, therefore, the subgroup $\left(\mathbf{S L}_{\mathbb{C}}(\mathbb{E})\right)^{\theta_{0}}=\mathbf{S L}_{\mathbb{C}}(\mathbb{E}) \cap \mathbf{U}(\mathbb{E})=G$.

The commuting involutive automorphisms $\sigma_{0}$ and $\sigma_{1}$ of $\mathfrak{g l}_{\mathbb{C}}(\mathbb{E})$ preserve the center $\mathbb{C} E$ and the commutant $\mathfrak{s l}_{\mathbb{C}}(\mathbb{E})$, which allows us to decompose this algebra into a direct sum of $\sigma_{1}$-invariant subspaces $\mathbb{C} E,\left(\mathfrak{s l}_{\mathbb{C}}(\mathbb{E})\right)^{\sigma_{0}}$, and $\left(\mathfrak{s l}_{\mathbb{C}}(\mathbb{E})\right)^{-\sigma_{0}}$. Furthermore, since $\left(\mathbf{S L}_{\mathbb{C}}(\mathbb{E})\right)^{\theta_{0}}=G \subset \mathbf{G L}_{\mathbb{C}}(\mathbb{E})$ we have that $\left(\mathfrak{s l}_{\mathbb{C}}(\mathbb{E})\right)^{\sigma_{0}}=\mathfrak{g} \subset \mathfrak{g l}_{\mathbb{C}}(\mathbb{E})$. Since $\mathbb{C}\left(\mathfrak{s l}_{\mathbb{C}}(\mathbb{E})\right)=$ $\mathfrak{s l}_{\mathbb{C}}(\mathbb{E}) \subset \mathfrak{g l}_{\mathbb{C}}(\mathbb{E})$, and $\sigma_{0}$ is an antilinear over $\mathbb{C}$ automorphism of $\mathfrak{g l}_{\mathbb{C}}(\mathbb{E})$, we have

$$
\left(\mathfrak{s l}_{\mathbb{C}}(\mathbb{E})\right)^{-\sigma_{0}}=i \cdot\left(\mathfrak{s l}_{\mathbb{C}}(\mathbb{E})\right)^{\sigma_{0}}=i \mathfrak{g} \subset \mathfrak{g l}_{\mathbb{C}}(\mathbb{E}) .
$$

By the above,

$$
V=\left(\mathfrak{g l}_{\mathbb{C}}(\mathbb{E})\right)^{-\sigma_{1}}=(\mathbb{C} E)^{-\sigma_{1}} \oplus \mathfrak{g}^{-\sigma_{1}} \oplus(i \mathfrak{g})^{-\sigma_{1}} \subset \mathfrak{g l}_{\mathbb{C}}(\mathbb{E})
$$


and $\left(\sigma_{1}-\operatorname{id}_{\mathfrak{g}}\right) \mathfrak{g}=\mathfrak{g}^{-\sigma_{1}} \subset \mathfrak{g}$. Since

$$
(\mathbb{C} E)^{-\sigma_{1}}=\mathbb{C} E \subset \mathfrak{g l}_{\mathbb{C}}(\mathbb{E}) \quad \text { and } \quad \sigma_{1} \in \operatorname{Aut}_{\mathbb{C}}\left(\mathfrak{g l}_{\mathbb{C}}(\mathbb{E})\right),
$$

we have that

$$
V=(\mathbb{C} E) \oplus \mathfrak{g}^{-\sigma_{1}} \oplus i\left(\mathfrak{g}^{-\sigma_{1}}\right) \subset \mathfrak{g l}_{\mathbb{C}}(\mathbb{E}) .
$$

It is clear that $v:=E \in V$. Moreover (see (4.2)),

$$
G_{v}=G^{\theta_{1}} \subset G, \quad \mathfrak{g} v=\left(\sigma_{1}-\mathrm{id}_{\mathfrak{g}}\right) \mathfrak{g}=\mathfrak{g}^{-\sigma_{1}} \subset \mathfrak{g},
$$

and the action $G_{v}: V$ is given by $g: \xi \rightarrow g \xi g^{-1}$. By (4.3), the representation $G_{v}: N_{v}$ is isomorphic to the direct sum of the trivial action $G^{\theta_{1}}: \mathbb{R}^{2}$ and the adjoint action $G^{\theta_{1}}: \mathfrak{g}^{-\sigma_{1}}$.

By Lemma 2.17, the quotient $\mathfrak{g}^{-\sigma_{1}} / G^{\theta_{1}}$ is homeomorphic to a closed half-space. Moreover, $N_{v} / G_{v} \cong \mathbb{R}^{2} \times\left(\mathfrak{g}^{-\sigma_{1}} / G^{\theta_{1}}\right)$ and, therefore, the quotient $N_{v} / G_{v}$ is also homeomorphic to a closed half-space.

Thus we have established that the quotient $N_{v} / G_{v}$ is not a manifold. By Lemma 2.1. neither is the quotient $V / G$.

Therefore, in each of cases (3) and (4) the quotient $V / G$ is not a manifold.

Now Theorem 1.2 is fully proved.

Theorems 1.1 and 1.2 immediately imply Corollary 1.3

\section{ACKNOWLEDGMENTS}

The author is grateful to Professor E. B. Vinberg for constant scientific support and numerous valuable advice.

\section{RefERENCES}

[1] O. G. Styrt, On the orbit space of a compact linear Lie group with a commutative connected component, Tr. Mosk. Mat. Obs. 70 (2009), 235-287; English transl., Trans. Moscow Math. Soc. 70 (2009), 171-206. MR2573640 (2011b:22019)

[2] O. G. Styrt, On the orbit space of a three-dimensional compact linear Lie groupIzv. Ross. Akad. Nauk Ser. Mat. 75 (2011), no. 4, 165-188; English transl., Izv. Math. 75 (2011), no. 4, 815-836. MR 2866189(2012m:22024)

[3] E. B. Vinberg and A. L. Onishchik, A seminar on Lie groups and algebraic groups, Second edition, URSS, Moscow, 1995. (Russian) MR1403378 (97d:22001)

[4] A. G. Elashvili, Canonical form and stationary subalgebras of points in general position for simple linear Lie groups, Funktsional. Anal. i Prilozhen 6 (1972), no. 1, 51-62. (Russian) MR0304554 $(46: 3689)$

[5] O. G. Styrt, On simplest isotropy subalgebras for compact linear Lie algebras, Tr. Mosk. Mat. Obs. 73 (2012) no. 1,133-150; English transl., Trans. Moscow Math. Soc. 73 (2012), 107-120.

[6] J. Dadok and V. Kac, Polar representations J. Algebra 92 (1985), no. 2, 504-524. MR778464 (86e:14023)

[7] P. K. Rashevsky, A theorem on the connectedness of the subgroup of a simply connected Lie group that commutes with one of its automorphisms Tr. Mosk. Mat. Obs. 30 (1974), 3-22. (Russian) MR.0463350 (57:3303)

[8] E. B. Vinberg and V. L. Popov, Invariant theory Algebraic geometry, 4 (Russian), 137-314, 315, Itogi Nauki i Tekhniki, Akad. Nauk SSSR, Vsesoyuz. Inst. Nauchn. i Tekhn. Inform., Moscow, 1989. (Russian) MR 1100485(92d:14010)

[9] E. B. Vinberg, The Weyl group of a graded Lie algebra, Izv. Akad. Nauk SSSR Ser. Mat. 40 (1976), no. 3, 488-526, 709. MR0430168 (55:3175)

Department of Mechanics and Mathematics, Moscow State University, Moscow

E-mail address: oleg_styrt@mail.ru 